\title{
Sulfoxide-Directed Enantioselective Synthesis of Functionalized Dihydropyrans
}

\author{
Roberto Fernández de la Pradilla, * and Mariola Tortosa
}

Instituto de Química Orgánica, CSIC, Juan de la Cierva, 3, 28006 Madrid, Spain.

Fax: 34-91-564-4853

E-mail: iqofp19@iqog.csic.es 


\section{MATERIALS AND METHODS}

Reagents and solvents were handled by using standard syringe techniques. All reactions were carried out under an argon atmosphere. Hexane, toluene and $\mathrm{CH}_{2} \mathrm{Cl}_{2}$ were distilled from $\mathrm{CaH}_{2}$, and THF and $\mathrm{Et}_{2} \mathrm{O}$ from sodium. $\mathrm{Et}_{3} \mathrm{~N}$, and $i-\mathrm{Pr}_{2} \mathrm{NH}$ were distilled from $\mathrm{CaH}_{2}$. Crude products were purified by flash chromatography on 230-400 mesh silica gel with distilled solvents. Analytical TLC was carried out on silica gel plates with detection by UV light, iodine, acidic vanillin solution, 10\% phosphomolybdic acid solution in ethanol. All reagents were commercial products. Organolithium reagents were titrated prior to use. ${ }^{1} \mathrm{NaH}$ and $\mathrm{KH}$ (60\% in mineral oil) were washed repeatedly with dry hexane and dried prior to use. Through this section, the volume of solvents is reported in $\mathrm{mL} / \mathrm{mmol} \mathrm{of}$ starting material. ${ }^{1} \mathrm{H}$ and ${ }^{13} \mathrm{C}$ NMR spectra were recorded at $200,300,400$ or $500 \mathrm{MHz}\left({ }^{1} \mathrm{H}\right)$ using $\mathrm{CDCl}_{3}$ as solvent and with the residual solvent signal as internal reference $\left(\mathrm{CDCl}_{3}, 7.24\right.$ and $\left.77.0 \mathrm{ppm}\right)$. The following abbreviations are used to describe peak patterns when appropriate: s (singlet), d (doublet), t (triplet), q (quartet), m (multiplet), br (broad), ap (apparent). Melting points are uncorrected. Optical rotations were measured at $20^{\circ} \mathrm{C}$ using a sodium lamp and in $\mathrm{CHCl}_{3}$ solution. Low resolution mass spectra were recorded using the electronic impact technique with an ionization energy of $70 \mathrm{eV}$ or using the atmospheric pressure chemical ionization (APCI) or electrospray (ES) chemical ionization techniques in its positive or negative modes. Elemental analyses were carried out at Instituto de Química Orgánica, CSIC (Madrid).

\section{General Procedure for the Synthesis of 3-Sulfinyl-3,6-dihydro-2H-pyrans.}

Method A: to a solution of the dienyl sulfoxide $(10 \mathrm{~mL} / \mathrm{mmol}$ of sulfoxide $)$ in $\mathrm{THF}$, at $-78^{\circ} \mathrm{C}$, under an argon atmosphere, was added 1.1 equiv of a freshly prepared 0.5 M LDA solution in THF. The mixture was allowed to warm to rt, monitored by TLC until completion (2-4 h) and quenched with a saturated solution of $\mathrm{NH}_{4} \mathrm{Cl}(3 \mathrm{~mL} / \mathrm{mmol}$ of sulfoxide) and water $(3 \mathrm{~mL} / \mathrm{mmol}$ of sulfoxide). The layers were separated and the aqueous phase was extracted with EtOAc $(5 \mathrm{~mL} / \mathrm{mmol}, 3$ times). The combined organic extracts were washed with a saturated solution of $\mathrm{NaCl}$, dried over anhydrous $\mathrm{MgSO}_{4}$ and filtered to give, after evaporation of the solvent, a crude product that was purified by chromatography on silica gel using the appropriate mixture of solvents.

Method B: to a suspension of 1.1-1.3 equiv of $\mathrm{NaH}$ or $\mathrm{KH}$ in $\mathrm{THF}$ ( $5 \mathrm{~mL} / \mathrm{mmol}$ of sulfoxide) at $-30{ }^{\circ} \mathrm{C}$, under an argon atmosphere, was added a solution of the dienyl sulfoxide in THF $(5 \mathrm{~mL} / \mathrm{mmol}$ of sulfoxide). The mixture was allowed to warm up to rt, monitored by TLC until completion (2-4 h) and quenched with a saturated solution of $\mathrm{NH}_{4} \mathrm{Cl}(3 \mathrm{~mL} / \mathrm{mmol}$ of sulfoxide $)$ and water $(3 \mathrm{~mL} / \mathrm{mmol} \mathrm{of}$ sulfoxide). The layers were separated and the aqueous phase was extracted with EtOAc $(5 \mathrm{~mL} / \mathrm{mmol}, 3$ times). The combined organic extracts were washed with a saturated solution of $\mathrm{NaCl}$, dried over 
anhydrous $\mathrm{MgSO}_{4}$ and filtered to give, after evaporation of the solvent a crude product that was purified by chromatography on silica gel using the appropriate mixture of solvents.

\subsection{Synthesis of (-)-(2S,3R, $\left.R_{S}\right)-2-n$-Butyl-3-(p-tolylsulfinyl)-3,6-dihydro-2H-pyran, $2 \mathrm{a}$.}

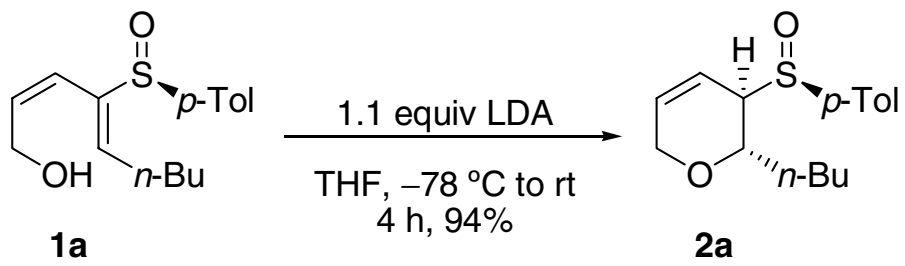

From dienyl sulfoxide 1a $(20 \mathrm{mg}, 0.070 \mathrm{mmol})$ and LDA $(0.15 \mathrm{~mL}, 0.077 \mathrm{mmol})$ following general procedure A (4 h), dihydropyran 2a was obtained. Purification by chromatography (10-50\% EtOAc-hexane) afforded $18 \mathrm{mg}(0.066 \mathrm{mmol}, 94 \%)$ of $\mathbf{2 a}$ as a colorless oil.

Data for 2a: $\boldsymbol{R}_{f}=0.34\left(50 \%\right.$ EtOAc-hexane). $[\alpha]^{\mathbf{2 0}}{ }_{\mathbf{D}}=-218.8(c=0.75) .{ }^{1} \mathbf{H}$ NMR (300 MHz)COSY $\delta 0.88(\mathrm{t}, 3 \mathrm{H}, J=7.1 \mathrm{~Hz}, \mathrm{Me}-n-\mathrm{Bu}), 1.25-1.54(\mathrm{~m}, 5 \mathrm{H}), 1.74(\mathrm{~m}, 1 \mathrm{H}), 2.39(\mathrm{~s}, 3 \mathrm{H}, \mathrm{Me}-p-$ Tol), 3.07 (m, 1 H, H-3), 4.09 (m, 2 H, H-6), 4.32 (dm, 1 H, $J=9.5$ Hz, H-2), 5.14 (ddd, 1 H, $J=10.2$, 4.9, $2.5 \mathrm{~Hz}, \mathrm{H}-4), 5.95$ (dtd, $1 \mathrm{H}, J=10.2,2.5,1.0 \mathrm{~Hz}, \mathrm{H}-5), 7.29$ (d, $2 \mathrm{H}, J=7.8 \mathrm{~Hz}, p$-Tol), 7.53 (d, $2 \mathrm{H}, J=8.3 \mathrm{~Hz}, p$-Tol). NOESY-1D between $\mathrm{H}-2 / \mathrm{CH}_{2}-n$-Bu: $0.8 \%$; between $\mathrm{H}-2 / p$-Tol: $0.8 \%$; between $\mathrm{H}-2 / \mathrm{H}-3$ : $1.5 \%$; between $\mathrm{H}-3 / p$-Tol: 1.6\%; between $\mathrm{H}-3 / \mathrm{H}-4: 1.4 \%$; between $\mathrm{H}-5 / \mathrm{H}-4: 1.5 \%$; between H-5/H-6: 2.6\%; between $\mathrm{H}-6 / \mathrm{H}-5$ : $2.3 \%$; between $\mathrm{H}-6 / \mathrm{CH}_{2}-n-\mathrm{Bu}: 1.0 \%$. ${ }^{13} \mathbf{C}$ NMR (50 MHz)-HSQC $\delta 14.0(\mathrm{Me}-n-\mathrm{Bu}), 21.5$ (Me-p-Tol), 22.5, 27.9, 30.7, 60.8 (C-6), 66.1 (C-3), 69.9 (C2), 116.6 (C-4), 125.5 (2 C), 129.7 (2 C), 132.6 (C-5), 138.9, 142.0. IR (film): 3038, 2932, 2857, 1651, 1596, 1493, 1462, 1380, 1302, 1210, 1182, 1082, 1037, 1016, $812 \mathrm{~cm}^{-1}$. MS (ES): 579 $[2 \mathrm{M}+\mathrm{Na}]^{+}, 301[\mathrm{M}+\mathrm{Na}]^{+}, 279[\mathrm{M}+1]^{+}(100 \%)$.

\subsection{Synthesis of (-)-(2S,3R, $\left.R_{S}\right)-2$-Phenyl-3-(p-tolylsulfinyl)-3,6-dihydro-2H-pyran, $2 \mathrm{~b}$.}<smiles>O=S([O-])C(/C=C\CO)=C/Pc1ccccc1</smiles>

$1 b$

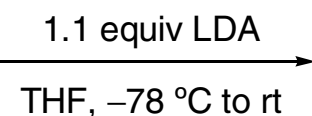
$3 \mathrm{~h}, 84 \%$

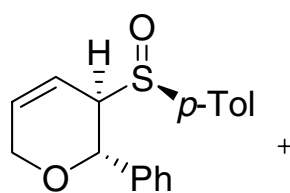

$\mathbf{2 b}(93)$<smiles>OC/C=C\C#CPc1ccccc1</smiles>

2b' (7)

From dienyl sulfoxide $\mathbf{1 b}(597 \mathrm{mg}, 2 \mathrm{mmol})$ and LDA $(4.4 \mathrm{~mL}, 2.2 \mathrm{mmol})$ following general procedure A (4 h), dihydropyran $\mathbf{2 b}$ was obtained. Purification by chromatography (15-50\% EtOAchexane) afforded $35 \mathrm{mg}(0.11 \mathrm{mmol}, 5 \%)$ of $\mathbf{2 b}$ ' as a colorless oil and $500 \mathrm{mg}(1.67 \mathrm{mmol}, 84 \%)$ of $\mathbf{2 b}$ as a pale yellow solid that was recrystallized from EtOAc-hexane. 
Data for 2b: $\mathbf{m p}: 87-89{ }^{\circ} \mathrm{C} . \quad \boldsymbol{R}_{f}=0.34\left(50 \%\right.$ EtOAc-hexane). $[\alpha]^{20}{ }_{\mathbf{D}}=-291.7(c=0.82) .{ }^{1} \mathbf{H}$ NMR (300 MHz)-COSY $\delta 2.44$ (s, 3 H, Me-p-Tol), 3.78 (m, 1 H, H-3), 3.91 (dq, 1 H, J = 17.6, 2.4 Hz, H-6), 4.14 (dm, 1 H, $J=17.6$ Hz, H-6), 5.33 (d, 1 H, $J=2.9$ Hz, H-2), 5.51 (ddtd, 1 H, $J=10.2$, 4.9, 2.2, 0.5 Hz, H-4), 6.00 (dtd, 1 H, $J=10.2,3.2,1.0 \mathrm{~Hz}, \mathrm{H}-5), 7.30-7.40$ (m, 7 H), 7.59 (d, 2 H, $J=$ $8.3 \mathrm{~Hz}$ ). NOESY-1D between $\mathrm{H}-2 / p$-Tol: $0.6 \%$; between $\mathrm{H}-2 / \mathrm{Ph}: 1.9 \%$; between $\mathrm{H}-2 / \mathrm{H}-3: 1.0 \%$; between $\mathrm{H}-3 / \mathrm{H}-2$ : 1.3\%; between $\mathrm{H}-3 / \mathrm{H}-4: 1.6 \%$; between $\mathrm{H}-3 / p$-Tol: 1.3\%; H-3/Ph: 3.2\%; between H-4/H-5: 2.2\%; between H-4/p-Tol: 1.1\%; between H-4/H-3: 1.5\%; between H-5/H-4: 1.3\%; between H-5/H-6\%: 1.1\%; between H-5/H-6 (4.14 ppm): 1.1\%; between H-5/H-6 (3.91 ppm): 1.9\%; between H-6/H-5: 2.1\%. ${ }^{13}$ C NMR (75 MHz)-HSQC $\delta 21.5$ (Me-p-Tol), 61.6 (C-6), 64.3 (C-3), 71.5 (C-2), 117.1 (C-4), 125.6 (2 C), 127.9 (2 C), 128.3, 128.5 (2 C), 129.7 (2 C), 133.2 (C-5), 137.3, 138.4, 142.2. IR (KBr): 2945, 2829, 1632, 1490, 1449, 1304, 1198, 1074, 1041, 1015, 960, 897, 821, 807, 752, $700 \mathrm{~cm}^{-1}$. MS (ES): $619[2 \mathrm{M}+\mathrm{Na}]^{+}, 321[\mathrm{M}+\mathrm{Na}]^{+}, 299[\mathrm{M}+1]^{+}(100 \%)$. Anal. calcd for $\mathrm{C}_{18} \mathrm{H}_{18} \mathrm{O}_{2} \mathrm{~S}: \mathrm{C}, 72.45 ; \mathrm{H}, 6.08 ; \mathrm{S}, 10.75$. Found: C,72.60; H, 7.26; S, 10.38 .

Data for 2b': $\boldsymbol{R}_{f}=0.45$ (50\% EtOAc-hexane). ${ }^{1} \mathbf{H}$ NMR (300 MHz) $\delta 1.61$ (br s, $\left.1 \mathrm{H}, \mathrm{OH}\right)$, 4.49 (t, 2 H, J = 4.6 Hz, H-1), 5.80 (dt, $1 \mathrm{H}, J=11.0,1.5 \mathrm{~Hz}, \mathrm{H}-3), 6.13$ (dt, $1 \mathrm{H}, J=11.0,6.3 \mathrm{~Hz}, \mathrm{H}-$ 2), 7.28-7.32 (m, 3 H, Ph), 7.40-7.43 (m, $2 \mathrm{H}, \mathrm{Ph})$. MS (ES): $159[\mathrm{M}+1]^{+}(100 \%), 141.0[(\mathrm{M}-18)+1]^{+}$.

\subsection{Synthesis of (-)-(2S,3R,6S, $\left.R_{S}\right)-6$-Methyl-2-phenyl-3-(p-tolylsulfinyl)-3,6-dihydro-2H-pyran,} 2c.
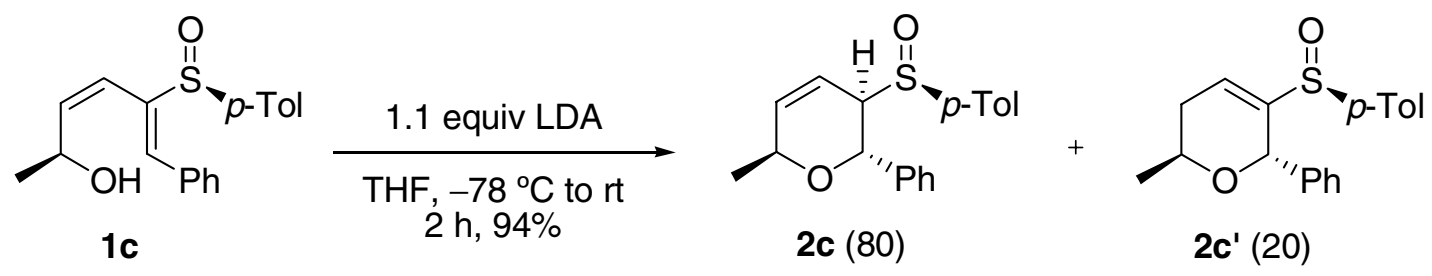

From dienyl sulfoxide 1c $(18 \mathrm{mg}, 0.060 \mathrm{mmol})$ and LDA $(0.13 \mathrm{~mL}, 0.066 \mathrm{mmol})$, following general procedure A (2 h), an 80:20 mixture of $\mathbf{2 c}$ and $\mathbf{2 c}$ ' was obtained. Purification by chromatography (10-50\% EtOAc-hexane) afforded $13 \mathrm{mg}(0.042 \mathrm{mmol}, 70 \%)$ of $2 \mathrm{c}$ and $3 \mathrm{mg}(0.010$ mmol, 17\%) of $\mathbf{2} \mathbf{c}^{\prime}$ both as white solids that were recrystallized from EtOAc-hexane.

Data for 2c: $\mathbf{m p}: 128-130{ }^{\circ} \mathrm{C} . \quad \boldsymbol{R}_{f}=0.21$ (30\% EtOAc-hexane). $[\alpha]^{20}{ }_{\mathbf{D}}=-237.1(c=0.73) .{ }^{1} \mathbf{H}$ NMR (400 MHz)-COSY $\delta 1.19$ (d, 3 H, $J=6.8$ Hz, Me-6), 2.42 (s, 3 H, Me-p-Tol), 3.66 (m, 1 H, H3), 3.88 (m, 1 H, H-6), 5.30 (dddd, 1 H, $J=10.4,5.5,2.2,1.1$ Hz, H-4), 5.58 (s, 1 H, H-2), 5.84 (dm, 1 $\mathrm{H}, J=10.2 \mathrm{~Hz}, \mathrm{H}-5), 7.27-7.37(\mathrm{~m}, 7 \mathrm{H}), 7.61(\mathrm{~d}, 2 \mathrm{H}, J=8.2 \mathrm{~Hz})$. NOESY-1D between H-3/H-2: 1.5\%; between $\mathrm{H}-3 / \mathrm{H}-4$ : 1.6\%; between $\mathrm{H}-3 / \mathrm{Ph}$ : 3.5\%; between $\mathrm{H}-3 / p$-Tol; between $\mathrm{H}-6 / \mathrm{H}-5$ : $1.6 \%$; 
between H-6/Me: 2.5\%; between H-6/Ph: 1.0\%; between Me/H-6: 1.2\%; Me/H-5: 0.5\%. ${ }^{13}$ C NMR (75 MHz)-HSQC $\delta 20.3$ (Me-6), 21.5 (Me-p-Tol), 64.3 (C-3), 65.1 (C-6), 70.9 (C-2), 116.3 (C-4), 125.5 (2 C), 127.8 (2 C), 128.1, 128.3 (2 C), 129.7 (2 C), 137.6, 138.1 (C-5), 138.9, 142.1. IR (KBr): 3057, 2971, 2920, 2833, 1635, 1595, 1490, 1452, 1085, 1068, 1049, 806, 747, $710 \mathrm{~cm}^{-1}$. MS (ES): $647[2 \mathrm{M}+\mathrm{Na}]^{+}(100 \%), 335[\mathrm{M}+\mathrm{Na}]^{+}, 313[\mathrm{M}+1]^{+}$. Anal. calcd for $\mathrm{C}_{19} \mathrm{H}_{20} \mathrm{O}_{2} \mathrm{~S}: \mathrm{C}, 73.04 ; \mathrm{H}, 6.45 ; \mathrm{S}$, 10.26. Found: C, 73.18; H, 6.37; S, 10.50 .

Data for 2c': $\boldsymbol{R}_{f}=0.16\left(30 \%\right.$ EtOAc-hexane). ${ }^{1} \mathbf{H}$ NMR $(200 \mathbf{M H z}) \delta 1.08(\mathrm{t}, 3 \mathrm{H}, J=6.2 \mathrm{~Hz}$, Me-6), 2.17 (ddm, $1 \mathrm{H}, J=18.1,9.1 \mathrm{~Hz}, \mathrm{H}-5$ ), 2.36 (s, $3 \mathrm{H}, \mathrm{Me}-p$-Tol), 2.46 (dm, $1 \mathrm{H}, J=18.1 \mathrm{~Hz}, \mathrm{H}-$ 5), 3.70 (m, 1 H, H-6), 4.71 (s, 1 H, H-2), 7.01 (m, 1 H, H-4), 7.19-7.59 (m, 9 H).

\subsection{Synthesis of $\left(2 S, 3 R, 6 R, R_{S}\right)-6$-Methyl-2-phenyl-3-(p-tolylsulfinyl)-3,6-dihydro-2H-pyran, 2 d.}

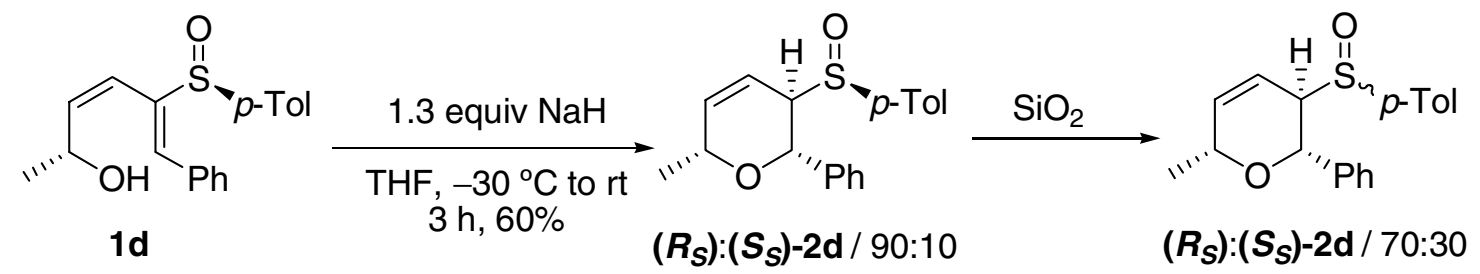

From dienyl sulfoxide 1d (10 mg, $0.032 \mathrm{mmol})$ and $\mathrm{NaH}$ (1 mg, $0.042 \mathrm{mmol}, 1.3 \mathrm{equiv})$, following general procedure B (2 h), a 90:10 mixture of $\left(\boldsymbol{R}_{S}\right)-\mathbf{2 d}$ and $\left(\boldsymbol{S}_{S}\right)$-2d was obtained. Purification by chromatography (10-50\% EtOAc-hexane) afforded $6 \mathrm{mg}(0.019,60 \%)$ of a 70:30 mixture of $\left(\boldsymbol{R}_{S}\right)$ and $\left(\boldsymbol{S}_{S}\right)$-2d. Oxidation of this mixture gave a single sulfone (see below).

Data for $\left(\boldsymbol{R}_{s}\right)$-2d (from the mixture): $\boldsymbol{R}_{f}=0.36$ (50\% EtOAc-hexane). ${ }^{1} \mathbf{H}$ NMR (300 MHz) $\delta$ 1.17 (d, 3 H, J = 6.6 Hz, Me-6), 2.38 (s, 3 H, Me-p-Tol), 4.10 (m, 2 H, H-3, H-6), 4.24 (d, 1 H, $J=9.3$ Hz, H-2), 5.95 (d, $1 \mathrm{H}, J=10.2 \mathrm{~Hz}), 6.12(\mathrm{~d}, 1 \mathrm{H}, J=10.2 \mathrm{~Hz}), 7.21-7.43(\mathrm{~m}, 9 \mathrm{H}) .{ }^{13} \mathbf{C}(\mathbf{5 0} \mathbf{M H z}$, partial data) $\delta 20.9,21.5,63.6,71.3,75.7,118.0,125.8,127.9,128.8,129.4$.

Data for $\left(\boldsymbol{S}_{s}\right)$-2d (from the mixture): $\boldsymbol{R}_{f}=0.33$ (50\% EtOAc-hexane). ${ }^{1} \mathbf{H}$ NMR (300 MHz) $\delta$ 1.26 (d, 3 H, $J=6.8$ Hz, Me-6), 2.38 (s, 3 H, Me-p-Tol), 3.35 (m, 1 H, H-3), 4.45 (m, 1 H, H-6), 4.92 (d, $1 \mathrm{H}, J=10.0 \mathrm{~Hz}, \mathrm{H}-2), 5.44$ (dm, $1 \mathrm{H}, J=10.5 \mathrm{~Hz}, \mathrm{H}-4), 6.09$ (dm, $1 \mathrm{H}, J=10.2 \mathrm{~Hz}, \mathrm{H}-5), 7.20$ $7.46(\mathrm{~m}, 7 \mathrm{H}), 7.55(\mathrm{~d}, 1 \mathrm{H}, J=6.6 \mathrm{~Hz})$.

\subsection{Synthesis of (2S,3R)-4-Methyl-2-phenyl-3-(p-tolylsulfinyl)-3,6-dihydro-2H-pyran, 2 e.}


S6

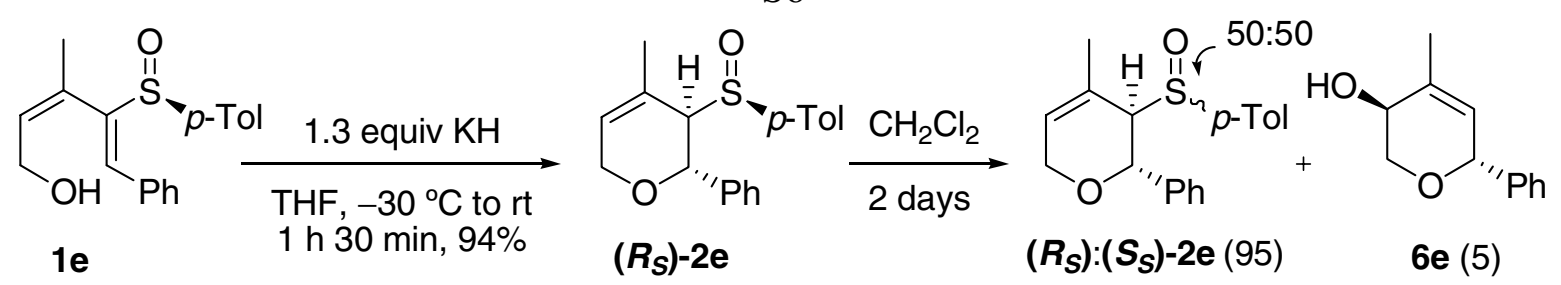

From dienyl sulfoxide 1e (15 mg, $0.048 \mathrm{mmol})$ and $\mathrm{KH}(2.5 \mathrm{mg}, 0.062 \mathrm{mmol}, 1.3 \mathrm{equiv})$ following general procedure B (1 h, $30 \mathrm{~min})$, dihydropyran $\mathbf{2 e}$ was obtained. The crude product was filtered through a short pad of silica gel $\left(20 \%\right.$ EtOAc- $\left.\mathrm{CH}_{2} \mathrm{Cl}_{2}\right)$ to give $13 \mathrm{mg}(0.042 \mathrm{mmol}, 87 \%)$ of $\mathbf{2 e}$ as a colorless oil. When compound $\mathbf{2 e}$ was left at $\mathrm{rt}$ for 2 days diastereomerization at sulfur took place affording a 50:50 mixture of $R_{s}: S_{S}$ dihydropyrans along with a small amount of alcohol 6e. Oxidation of this mixture gave a single sulfone (see below).

Data for 2e: $\mathbf{m p}: 91-93{ }^{\circ} \mathrm{C} . \quad \boldsymbol{R}_{f}=0.32\left(20 \%\right.$ EtOAc- $\left.\mathrm{CH}_{2} \mathrm{Cl}_{2}\right) .{ }^{1} \mathbf{H} \mathbf{N M R}(300 \mathrm{MHz}) \delta 1.39(\mathrm{q}$, $3 \mathrm{H}, J=1.9 \mathrm{~Hz}, \mathrm{Me}-4), 2.39$ (s, $3 \mathrm{H}, \mathrm{Me}-p$-Tol), 3.61 (m, $1 \mathrm{H}, \mathrm{H}-3$ ), 3.89 (dsext, $1 \mathrm{H}, J$ = 17.1, 2.2 Hz, H-6), 4.17 (dm, $1 \mathrm{H}, J=17.3 \mathrm{~Hz}, \mathrm{H}-6), 5.55$ (d, $1 \mathrm{H}, J=2.2 \mathrm{~Hz}, \mathrm{H}-2$ ), 5.63 (oct, $1 \mathrm{H}, J=1.5 \mathrm{~Hz}, \mathrm{H}-5$ ), 7.18-7.30 (m, $7 \mathrm{H}), 7.58(\mathrm{~d}, 2 \mathrm{H}, J=8.3 \mathrm{~Hz}) . \quad \mathbf{M S}(\mathrm{ES}): 647[2 \mathrm{M}+\mathrm{Na}]^{+}, 335[\mathrm{M}+\mathrm{Na}]^{+}, 313[\mathrm{M}+1]^{+}$ (100\%). Anal. calcd for $\mathrm{C}_{19} \mathrm{H}_{20} \mathrm{O}_{2} \mathrm{~S}: \mathrm{C}, 73.04 ; \mathrm{H}, 6.45 ; \mathrm{S}, 10.26$. Found: $\mathrm{C}, 73.12 ; \mathrm{H}, 6.27 ; \mathrm{S}, 10.35$.

Data for $\left(\boldsymbol{S}_{s}\right)$-2e (from the mixture): $\boldsymbol{R}_{f}=0.34\left(20 \%\right.$ EtOAc- $\left.\mathrm{CH}_{2} \mathrm{Cl}_{2}\right),{ }^{1} \mathbf{H} \mathbf{N M R}(300 \mathrm{MHz}) \delta$ 2.05 (s, 3 H, Me-4), 2.39 (s, 3 H, Me-p-Tol), 3.64 (m, 1 H, H-3), 3.84 (m, 2 H, H-6), 5.22 (s, 1 H, H-2), $5.70(\mathrm{~m}, 1 \mathrm{H}, \mathrm{H}-5), 7.20-7.30(\mathrm{~m}, 7 \mathrm{H}), 7.51(\mathrm{dm}, 2 \mathrm{H}, J=8.3 \mathrm{~Hz})$.

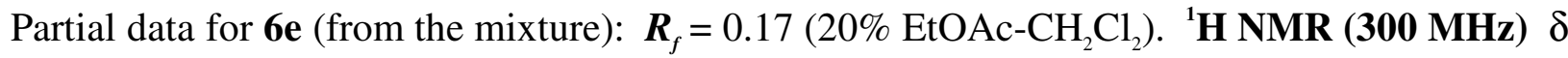
$1.91(\mathrm{t}, 3 \mathrm{H}, J=1.7 \mathrm{~Hz}, \mathrm{Me}-4), 3.63(\mathrm{dd}, 1 \mathrm{H}, J=12.4,5.1 \mathrm{~Hz}, \mathrm{H}-2), 3.88(\mathrm{dd}, 1 \mathrm{H}, J=12.7,3.7 \mathrm{~Hz}$, H-2), 3.90 (m, $1 \mathrm{H}, J=12.7,3.7 \mathrm{~Hz}, \mathrm{H}-2), 5.15$ (m, $1 \mathrm{H}, \mathrm{H}-6), 5.74$ (m, $1 \mathrm{H}, \mathrm{H}-5)$.

\subsection{Synthesis of (-)-(2R,3R, $\left.R_{S}\right)-2-n$-Butyl-3-(p-tolylsulfinyl)-3,6-dihydro-2H-pyran, 4 a.}

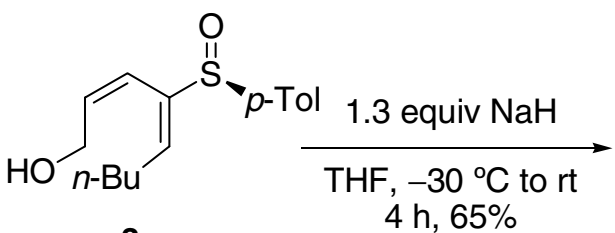

3a

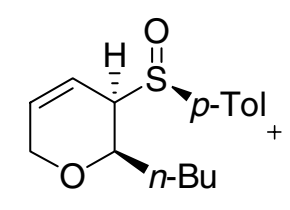

4a (96)

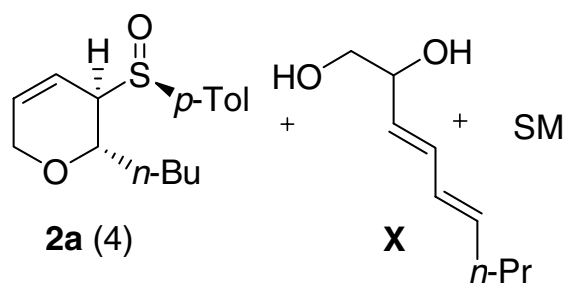

$2 a(4)$

From dienyl sulfoxide 3a (20 mg, $0.070 \mathrm{mmol})$ and $\mathrm{NaH}(2.2 \mathrm{mg}, 0.090 \mathrm{mmol}, 1.3 \mathrm{equiv})$ following general procedure $\mathbf{B}(2 \mathrm{~h})$, a 65:3:16:16 mixture of $\mathbf{4 a}, \mathbf{2 a}, \mathbf{X}$ and starting material was obtained. Purification by chromatography (10-50\% EtOAc-hexane) afforded $13 \mathrm{mg}$ (0.048 mmol, $65 \%$ ) of $\mathbf{4 a}$ as a white solid that was recrystallized from $\mathrm{Et}_{2} \mathrm{O}$-hexane and $6 \mathrm{mg}$ of an inseparable mixture of diol $\mathbf{X}$ and starting material. 

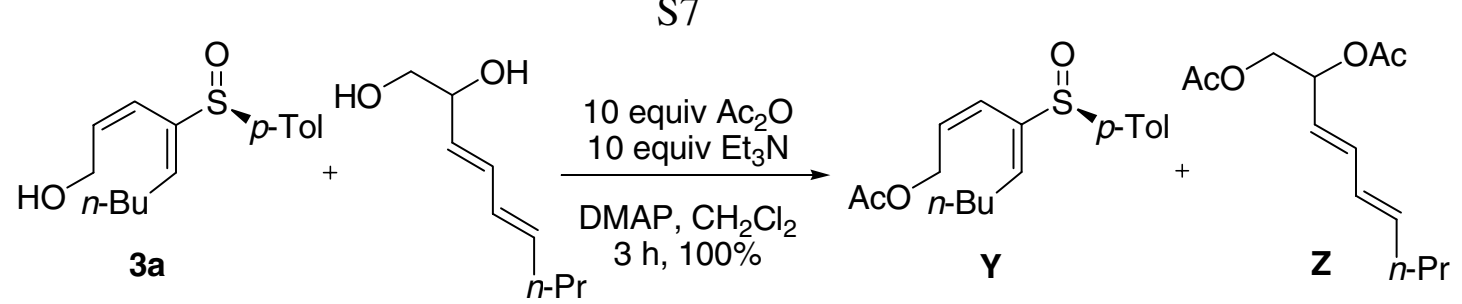

To a solution of $5 \mathrm{mg}$ of a 50:50 mixture of $\mathbf{3 a}$ and $\mathbf{X}$ in $0.5 \mathrm{~mL}$ of $\mathrm{CH}_{2} \mathrm{Cl}_{2}$, was added acetic anhydride ( $30 \mu \mathrm{L}, 0.32 \mathrm{mmol}, 10$ equiv), $\mathrm{Et}_{3} \mathrm{~N}(50 \mu \mathrm{L}, 0.32 \mathrm{mmol}, 10$ equiv) and a catalytic amount of DMAP. The mixture was stirred at $\mathrm{rt}$ for $3 \mathrm{~h}$, quenched with a saturated solution of $\mathrm{NH}_{4} \mathrm{Cl}$ and water and diluted with $\mathrm{CH}_{2} \mathrm{Cl}_{2}$. The layers were separated and the aqueous phase was extracted twice with $\mathrm{CH}_{2} \mathrm{Cl}_{2}$. The combined organic extracts were washed with a saturated solution of $\mathrm{NaCl}$, dried over $\mathrm{MgSO}_{4}$ and the solvent was evaporated. Purification by chromatography (5-50\% EtOAc-hexane) afforded $5 \mathrm{mg}(0.016 \mathrm{mmol}, 50 \%)$ of $\mathbf{Y}$ and $4 \mathrm{mg}(0.016 \mathrm{mmol}, 50 \%)$ of diacetate $\mathbf{Z}$ both as colorless oils.

Data for 4a: $\mathbf{m p}: 80-82{ }^{\circ} \mathrm{C} . \quad \boldsymbol{R}_{f}=0.33$ (50\% EtOAc-hexane). $[\alpha]^{20}{ }_{\mathbf{D}}=-334.1(c=0.91) .{ }^{1} \mathbf{H}$ NMR (300 MHz)-COSY $\delta 0.93$ (t, $3 \mathrm{H}, J=7.1 \mathrm{~Hz}, \mathrm{Me}-n-\mathrm{Bu}), 1.33-1.64$ (m, $4 \mathrm{H}), 1.99-2.07$ (m, 2 H), 2.39 (s, 3 H, Me-p-Tol), 3.30 (dqd, 1 H, $J=5.9,2.2,1.0 \mathrm{~Hz}, \mathrm{H}-3$ ), 3.83 (ddd, $1 \mathrm{H}, J=6.8,5.8,2.2$ Hz, H-2), 4.15 (dd, 2 H, $J=4.6,2.3 \mathrm{~Hz}, \mathrm{H}-6$ ), 5.02 (ddt, $1 \mathrm{H}, J=10.2,5.9,2.2, \mathrm{H}-4$ ), 5.76 (dtd, $1 \mathrm{H}, J$ $=10.0,2.5,1.0 \mathrm{~Hz}, \mathrm{H}-5), 7.26$ (d, $2 \mathrm{H}, J=8.5 \mathrm{~Hz}, p$-Tol), 7.53 (d, $2 \mathrm{H}, J=8.3 \mathrm{~Hz}, p$-Tol). NOESY1D between $\mathrm{H}-2 / \mathrm{H}-3$ : $2.2 \%$; between $\mathrm{H}-2 / \mathrm{H}-6$ : $1.9 \%$. between $\mathrm{H}-3 / \mathrm{H}-2$ : $2.3 \%$; between $\mathrm{H}-3 / \mathrm{H}-4$ : 2.0\%; between $\mathrm{H}-3 / \mathrm{CH}_{2}-n-\mathrm{Bu}: 1.6 \%$; between $\mathrm{CH}_{2}-n-\mathrm{Bu} / \mathrm{H}-3: 0.9 \%$; between $\mathrm{CH}_{2}-n-\mathrm{Bu} / \mathrm{H}-2: 1.2 \%$; between $\mathrm{CH}_{2}-n$-Bu/p-Tol: 0.3\%. ${ }^{13} \mathrm{C}$ NMR (75 MHz)-HSQC $\delta 14.0(\mathrm{Me}-n$-Bu), $21.4(\mathrm{Me}-p$-Tol), $22.5\left(\mathrm{CH}_{2} n\right.$-Bu), $28.7\left(\mathrm{CH}_{2} n-\mathrm{Bu}\right), 32.8\left(\mathrm{CH}_{2} n\right.$-Bu), $66.6(\mathrm{C}-3), 66.8$ (C-6), 75.8 (C-2), 120.1 (C-4), 126.0 (2 C), 129.4 (2 C), 131.4 (C-5), 139.1, 141.6. IR (KBr): 3043, 2956, 2927, 2847, 1631, 1261 , 1184, 1091, 1042, $812 \mathrm{~cm}^{-1}$. MS (ES): $579[2 \mathrm{M}+\mathrm{Na}]^{+}, 301[\mathrm{M}+\mathrm{Na}]^{+}, 279[\mathrm{M}+1]^{+}(100 \%)$. Anal. calcd for $\mathrm{C}_{16} \mathrm{H}_{22} \mathrm{O}_{2} \mathrm{~S}: \mathrm{C}, 69.02 ; \mathrm{H}, 7.96 ; \mathrm{S}, 11.52$. Found: C, 69.14; H, 7.73; S, 11.66.

Data for diacetate Z: $\boldsymbol{R}_{f}=0.18$ (15\% EtOAc-hexane). ${ }^{1} \mathbf{H} \mathbf{N M R}(\mathbf{3 0 0} \mathbf{~ M H z}) \delta 0.88(\mathrm{t}, 3 \mathrm{H}, J$ = $7.3 \mathrm{~Hz}, \mathrm{Me}-n-\mathrm{Pr}$ ), 1.39 (sext, $2 \mathrm{H}, J=7.3 \mathrm{~Hz}, \mathrm{H}-8$ ), 2.04 (s, $3 \mathrm{H}, \mathrm{Me}-\mathrm{CO}$ ), 2.05 (m, $2 \mathrm{H}, \mathrm{H}-7$ ), 2.07 (s, $3 \mathrm{H}, \mathrm{Me}-\mathrm{CO}$ ), 4.07 (dd, $1 \mathrm{H}, J=11.7,7.1 \mathrm{~Hz}, \mathrm{H}-1$ ), 4.21 (dd, $1 \mathrm{H}, J=11.7,3.4 \mathrm{~Hz}, \mathrm{H}-1$ ), 5.41-5.52 (m, 2 H, H-3, H-2), 5.75 (dt, $1 \mathrm{H}, J=15.1,7.1 \mathrm{~Hz}, \mathrm{H}-6$ ), 5.99 (ddt, $1 \mathrm{H}, J=15.1,10.3,1.2 \mathrm{~Hz}, \mathrm{H}-5$ ), $6.28(\mathrm{dd}, 1 \mathrm{H}, J=14.2,10.4 \mathrm{~Hz}, \mathrm{H}-4)$.

Partial data for acetate $\mathbf{Y}: \boldsymbol{R}_{f}=0.42\left(50 \%\right.$ EtOAc-hexane). ${ }^{1} \mathbf{H}$ NMR (300 MHz) $\delta 0.87$ (t, 3 H, $J=6.6 \mathrm{~Hz}, \mathrm{Me}-n-\mathrm{Bu}), 1.30$ (m, 2 H), 1.45 (m, 2 H), 1.99 (s, 3 H, Me-CO), 2.10 (m, 2 H), 2.39 (s, 3 H, Me-p-Tol), 4.22 (m, 2 H, H-1), 5.73 (dm, $1 \mathrm{H}, J=13.1 \mathrm{~Hz}, \mathrm{H}-3), 5.85$ (dt, $1 \mathrm{H}, J=13.1,6.5 \mathrm{~Hz}, \mathrm{H}-$ 2), 6.53 (td, $1 \mathrm{H}, J=7.6,1.2 \mathrm{~Hz}, \mathrm{H}-5)$. 
1.7. Synthesis of (+)-(2R,3S, $\left.R_{S}\right)$-2-Phenyl-3-(p-tolylsulfinyl)-3,6-dihydro-2H-pyran, $5 \mathrm{~b}$.<smiles>O=S([Te])C(/C=C\CO)=C/c1ccccc1</smiles>

$3 b$

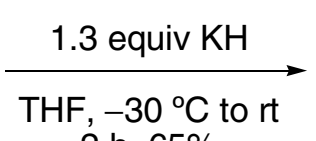

$2 \mathrm{~h}, 65 \%$

From dienyl sulfoxide $3 \mathbf{b}(30 \mathrm{mg}, 0.100 \mathrm{mmol})$ and $\mathrm{NaH}(5.2 \mathrm{mg}, 0.130 \mathrm{mmol}, 1.3$ equiv), following general procedure $\mathbf{B}(2 \mathrm{~h})$, a mixture of $\mathbf{5 b}$ and $\mathbf{5 b}$ ' was obtained. Purification by chromatography (20-50\% EtOAc-hexane) afforded $20 \mathrm{mg}(0.067 \mathrm{mmol}, 67 \%)$ of $\mathbf{5 b}$ and $3 \mathrm{mg}(0.010$ mmol, $10 \%$ ) of $\mathbf{5} \mathbf{b}$ ' both as colorless oils.

Data for 5b: $\quad \boldsymbol{R}_{f}=0.29$ (50\% EtOAc-hexane). $\quad[\alpha]^{20}{ }_{\mathbf{D}}=+248.2(c=0.50) . \quad{ }^{1} \mathbf{H}$ NMR (300 MHz)-COSY $\delta 2.37$ (s, $3 \mathrm{H}, \mathrm{Me}-p$-Tol), 3.45 (ap dquint, $1 \mathrm{H}, J=8.3,2.7 \mathrm{~Hz}, \mathrm{H}-3$ ), 4.19 (ddd, $1 \mathrm{H}, J$ = 17.1, 5.6, $2.5 \mathrm{~Hz}, \mathrm{H}-6), 4.32$ (ddd, $1 \mathrm{H}, J=17.1,5.5,2.3 \mathrm{~Hz}, \mathrm{H}-6), 4.90(\mathrm{~d}, 1 \mathrm{H}, J=8.5 \mathrm{~Hz}, \mathrm{H}-2)$, 5.59 (dq, $1 \mathrm{H}, J=10.5,2.3 \mathrm{~Hz}, \mathrm{H}-4), 6.20$ (ddt, $1 \mathrm{H}, J=10.5,3.4,2.1 \mathrm{~Hz}, \mathrm{H}-5), 7.26-7.45$ (m, $7 \mathrm{H}$ ), $7.50(\mathrm{dm}, 2 \mathrm{H}, J=8.3 \mathrm{~Hz})$. NOESY-1D between H-2/p-Tol: 2.3\%; between $\mathrm{H}-3 / \mathrm{H}-4: 2.3 \%$; between H-3/p-Tol: $1.8 \%$; between H-3/Ph: 2\%; between H-6/H-5: 5.7\%; between H-6/H-5: 2\%; between H6/H-2: 2.9\%. ${ }^{13} \mathrm{C}$ NMR (75 MHz)-HSQC $\delta 21.4$ (Me-p-Tol), 65.3 (C-6), 65.7 (C-3), 75.7 (C-2), 117.3 (C-4), 124.3 (2 C), 127.6 (2 C), 128.8 (3 C), 129.8 (2 C), 134.2 (C-5), 137.6, 138.8, 141.2. IR (film): 3028, 2923, 2855, 1596, 1492, 1453, 1177, 1084, 1045, 910, 809, $757 \mathrm{~cm}^{-1}$. MS (ES): 619 $[2 \mathrm{M}+\mathrm{Na}]^{+}(100 \%), 321[\mathrm{M}+\mathrm{Na}]^{+}, 299[\mathrm{M}+1]^{+}$.

Partial data for 5b': $\boldsymbol{R}_{f}=0.16\left(10 \%\right.$ EtOAc- $\left.\mathrm{CH}_{2} \mathrm{Cl}_{2}\right) . \quad{ }^{1} \mathbf{H} \mathbf{N M R}(\mathbf{3 0 0} \mathbf{M H z}) \delta 2.33(\mathrm{~s}, 3 \mathrm{H}$, Me-p-Tol), 2.45 (m, 2 H, H-5), 3.67 (ddd, $1 \mathrm{H}, J=11.6,6.3,5.1 \mathrm{~Hz}, \mathrm{H}-6$ ), 3.87 (dt, $1 \mathrm{H}, J=11.7,5.6$ Hz, H-6), 5.20 (ap dd, 1 H, $J=3.9,2.2 \mathrm{~Hz}, \mathrm{H}-2$ ), 6.85 (ddd, $1 \mathrm{H}, J=4.6,3.4,1.5 \mathrm{~Hz}, \mathrm{H}-4$ ), 7.08-7.22 $(\mathrm{m}, 9)$.

\section{General procedure for sulfoxide-sulfenate rearrangement of allyl sulfoxides.}

A kimble vial equipped with a stirring bar, was charged with a solution of the corresponding sulfoxide and 10 equiv of $\mathrm{P}(\mathrm{OMe})_{3}$ in $\mathrm{MeOH}$ and argon was bubbled through the solution for 10 min. The vial was quickly stoppered and inmersed in a pre-heated oil bath at $50-60{ }^{\circ} \mathrm{C}$ and monitored by TLC until completion. The solvent was removed under reduced pressure, and the residue was diluted with water and EtOAc. The layers were separated and the organic phase was washed with water $(2 \mathrm{~mL} /$ mmol of $\mathrm{P}(\mathrm{OMe})_{3}, 3$ times) and a saturated solution of $\mathrm{NaCl}$, dried over anhydrous $\mathrm{MgSO}_{4}$ and 
concentrated under reduced pressure to give a crude product that was purified by chromatography on silica gel using the appropriate mixture of solvents.

\subsection{Synthesis of (+)-(3S,6R)-6-Phenyl-3,6-dihydro-2H-pyran-3-ol, 6 b.}

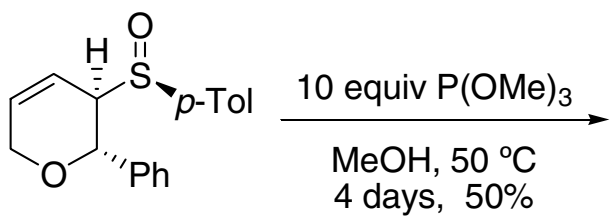

2b

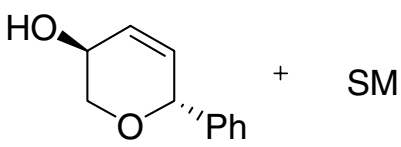

$6 b$

From sulfoxide $\mathbf{2 b}(15 \mathrm{mg}, 0.050 \mathrm{mmol})$ and $\mathrm{P}(\mathrm{OMe})_{3}(60 \mu \mathrm{L}, 0.500 \mathrm{mmol})$, following the general procedure (4 days) a 50:50 mixture of $\mathbf{6 b}$ and starting material was obtained along with minor products of unknown structure. Purification by chromatography (5-10\% EtOAc- $\left.\mathrm{CH}_{2} \mathrm{Cl}_{2}\right)$ afforded 12 $\mathrm{mg}$ of an inseparable mixture of $\mathbf{6 b}$ and $\mathbf{2 b}$. A second chromatography $\left(10 \% \mathrm{EtOAc}-\mathrm{CH}_{2} \mathrm{Cl}_{2}\right)$ gave 4 $\mathrm{mg}(0.023 \mathrm{mmol}, 46 \%)$ of pure $\mathbf{6 b}$ as a white solid and $8 \mathrm{mg}$ of a mixture of $\mathbf{6 b}$ and $\mathbf{2 b}$.

Data for 6b: $\mathbf{m p}: 50-52{ }^{\circ} \mathrm{C} . \quad \boldsymbol{R}_{f}=0.16(30 \%$ EtOAc-hexane $) . \quad[\alpha]^{20}{ }_{\mathbf{D}}=+179.5(c=0.20) .{ }^{1} \mathbf{H}$ NMR (300 MHz) $\delta 1.80(\mathrm{~d}, 1 \mathrm{H}, J=8.1 \mathrm{~Hz}, \mathrm{OH}), 3.58(\mathrm{dd}, 1 \mathrm{H}, J=11.5,5.4 \mathrm{~Hz}, \mathrm{H}-2), 3.99(\mathrm{dd}, 1$ $\mathrm{H}, J=11.5,4.4 \mathrm{~Hz}, \mathrm{H}-2), 4.04$ (br s, $1 \mathrm{H}, \mathrm{H}-3), 5.14$ (q, $1 \mathrm{H}, J=2.0 \mathrm{~Hz}, \mathrm{H}-6), 6.01$ (dm, $1 \mathrm{H}, J=10.2$ Hz, H-5), 6.08 (ddd, 1 H, $J=10.2,3.2,2.4$ Hz, H-6), 7.26-7.42 (m, 5 H). NOESY-1D between H-2 (3.58 ppm)/H-6: 0.6\%; between H-6/H-2 (3.58 ppm): 0.4\%; between H-6/Ph: $0.9 \%$, ${ }^{13}$ C NMR (50 MHz) $\delta$ 62.6, 68.0, 75.2, 127.7 (2 C), 128.2, 128.5 (3 C), 131.3, 139.4. IR (KBr): 3369, 2925, 2854, 1493, 1453, 1259, 1082, 903, 849, 810, $756 \mathrm{~cm}^{-1}$. MS (ES): $199[\mathrm{M}+\mathrm{Na}]^{+}, 159[(\mathrm{M}-18)+1]^{+}(100 \%)$. Anal. calcd for $\mathrm{C}_{11} \mathrm{H}_{12} \mathrm{O}_{2}: \mathrm{C}, 74.98 ; \mathrm{H}, 6.86$. Found: $\mathrm{C}, 74.63 ; \mathrm{H}, 7.05$.

\subsection{Synthesis of (-)-(3R,6S)-6-Phenyl-3,6-dihydro-2H-pyran-3-ol, ent-6b.}

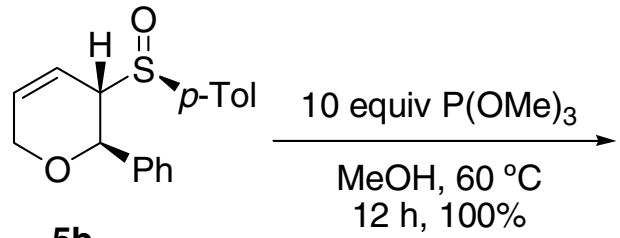

$5 b$

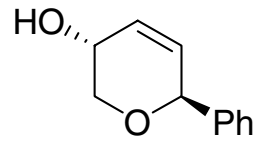

ent-6b

From sulfoxide $5 \mathbf{b}(10 \mathrm{mg}, 0.03 \mathrm{mmol})$ and $\mathrm{P}(\mathrm{OMe})_{3}(40 \mu \mathrm{L}, 0.30 \mathrm{mmol})$, following the general procedure (12 h), compound ent-6b was obtained. Purification by chromatography (15-30\% EtOAc-hexane $)$ afforded $6 \mathrm{mg}(0.03 \mathrm{mmol}, 100 \%)$ of $\mathbf{6 b}$ as a white solid that was recrystallized from $\mathrm{Et}_{2} \mathrm{O}$-hexane. 
Data for $\boldsymbol{e n t - 6 b}$ was identical to that described above for $\mathbf{6 b}$ except for optical rotation: $[\alpha]^{20}{ }_{\mathbf{D}}=$ $+150.4(c=0.73)$.

\section{Synthesis of $(3 R, 6 S)-6$-Phenyl-3,6-dihydro-2H-pyran-3-yl acetate,7b.}

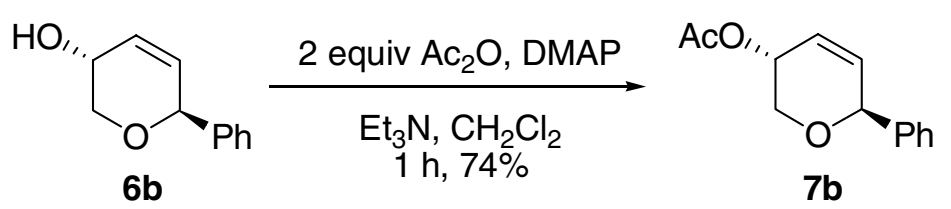

To a solution of $\mathbf{6 b}(3 \mathrm{mg}, 0.018 \mathrm{mmol})$ in $0.2 \mathrm{~mL}$ of $\mathrm{CH}_{2} \mathrm{Cl}_{2}$, was added acetic anhydride (9 $\mu \mathrm{L}, 0.09 \mathrm{mmol}, 5.0$ equiv), $\mathrm{Et}_{3} \mathrm{~N}(13 \mu \mathrm{L}, 0.09 \mathrm{mmol}, 5.0$ equiv) and a catalytic amount of DMAP. The mixture was stirred at $\mathrm{rt}$. for $1 \mathrm{~h}$, quenched with a saturated solution of $\mathrm{NH}_{4} \mathrm{Cl}(2 \mathrm{~mL} / \mathrm{mmol})$ and water ( $2 \mathrm{~mL} / \mathrm{mmol}$ ) and diluted with $\mathrm{CH}_{2} \mathrm{Cl}_{2}$. The layers were separated and the aqueous phase was extracted twice with $\mathrm{CH}_{2} \mathrm{Cl}_{2}$. The combined organic extracts were washed with a saturated solution of $\mathrm{NaCl}$, dried over $\mathrm{MgSO}_{4}$ and the solvent was evaporated. Purification by chromatography (10-30\% EtOAchexane) afforded $3 \mathrm{mg}(0.014 \mathrm{mmol}, 74 \%)$ of $\mathbf{7 b}$ as a colorless oil.

Data for 7b: $\boldsymbol{R}_{f}=0.56\left(50 \%\right.$ EtOAc-hexane). ${ }^{1} \mathbf{H}$ NMR (300 MHz) $\delta 2.09(\mathrm{~s}, 3 \mathrm{H}, \mathrm{Me}-\mathrm{CO})$, 3.67 (dd, $1 \mathrm{H}, J=11.8,5.1 \mathrm{~Hz}, \mathrm{H}-2), 4.03$ (dd, $1 \mathrm{H}, J=11.8,4.4 \mathrm{~Hz}, \mathrm{H}-2), 5.19$ (dd, $1 \mathrm{H}, J=4.4,2.2$ Hz, H-6), 5.26 (m, 1 H, H-3), 6.02 (dt, $1 \mathrm{H}, J=10.2,2.4 \mathrm{~Hz}$ ), 6.14 (ddd, $1 \mathrm{H}, J=10.2,2.5,1.1 \mathrm{~Hz}$ ), 7.30-7.36 (m, $5 \mathrm{H})$.

\section{Synthesis of (3S,6S)-6-Phenyl-3,6-dihydro-2H-pyran-3-yl acetate, $8 \mathrm{~b}$.}

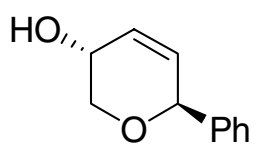

$6 b$

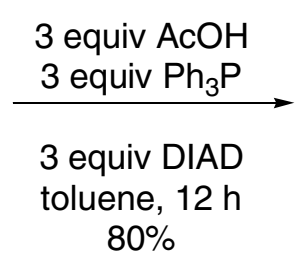

$80 \%$

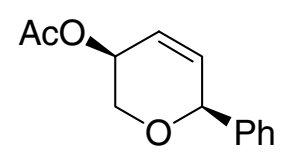

$8 b$

To a solution of $\mathbf{6 b}(6 \mathrm{mg}, 0.035 \mathrm{mmol}), \mathrm{Ph}_{3} \mathrm{P}(28 \mathrm{mg}, 0.105 \mathrm{mmol}, 3.0$ equiv) and acetic acid (6 $\mu \mathrm{L}, 0.105 \mathrm{mmol}, 3.0$ equiv) in toluene $(10 \mathrm{~mL} / \mathrm{mmol})$, under an argon atmosphere, was added diisopropyl azodicarboxylate $(20 \mu \mathrm{L}, 0.105 \mathrm{mmol}, 3.0$ equiv) dropwise. The reaction was monitored by TLC until starting material disappearance $(17 \mathrm{~h})$ and the solvent was removed under reduced pressure. Purification by chromatography (5-30\% EtOAc-hexane) afforded $6 \mathrm{mg}$ (0.023 mmol, 66\%) of $\mathbf{8 b}$ as a colorless oil.

Data for 8b: $\boldsymbol{R}_{f}=0.21$ (15\% EtOAc-hexane). ${ }^{1} \mathbf{H}$ NMR (200 MHz) $\delta 2.11(\mathrm{~s}, 3 \mathrm{H}, \mathrm{Me}-\mathrm{CO})$, 3.90 (dd, $1 \mathrm{H}, J=12.8,2.6 \mathrm{~Hz}, \mathrm{H}-2), 4.11$ (d, $1 \mathrm{H}, J=12.8 \mathrm{~Hz}, \mathrm{H}-2), 5.05$ (s, $1 \mathrm{H}, \mathrm{H}-6), 5.09$ (m, $1 \mathrm{H}$, H-3), 6.07 (m, 2 H, H-4, H-5), 7.32-7.36 (m, 5 H). 


\section{General Procedure for Oxidation of Sulfoxides.}

Oxidation with MMPP: to a cold $\left(0^{\circ} \mathrm{C}\right)$ solution of the sulfoxide in $\mathrm{MeOH}(10 \mathrm{~mL} / \mathrm{mmol})$ was added 1.5 equiv of magnesium monoperoxyphthalate hexahydrate (MMPP). The mixture was allowed to warm to rt, monitored by TLC until completion and then quenched with a saturated solution of $\mathrm{NaHCO}_{3}(4 \mathrm{~mL} / \mathrm{mmol})$. After removal of $\mathrm{MeOH}$ under reduced pressure, the mixture was diluted with EtOAc $(5 \mathrm{~mL} / \mathrm{mmol})$, the layers were separated and the aqueous phase was extracted with EtOAc (3 times, $5 \mathrm{~mL} / \mathrm{mmol})$. The combined organic layers were washed with a saturated solution of $\mathrm{NaCl}(1$ $\mathrm{mL} / \mathrm{mmol}$ ), dried over anhydrous $\mathrm{MgSO}_{4}$, filtered and concentrated under reduced pressure to give a crude product that was purified by chromatography on silica gel using the appropriate mixture of solvents.

Oxidation with $\boldsymbol{m}$-CPBA: to a solution of the sulfoxide in $\mathrm{CH}_{2} \mathrm{Cl}_{2}(10 \mathrm{~mL} / \mathrm{mmol})$ was added 3 equiv of $\mathrm{K}_{2} \mathrm{CO}_{3}$. The mixture was cooled to $0{ }^{\circ} \mathrm{C}$ and 1.5 equiv of $m$-CPBA was added. The solution was allowed to warm to rt, monitored by TLC until completion and then quenched with a $1 \mathrm{M}$ solution of $\mathrm{Na}_{2} \mathrm{~S}_{2} \mathrm{O}_{4}(2 \mathrm{~mL} / \mathrm{mmol})$ and $5 \%$ solution of $\mathrm{NaHCO}_{3}(2 \mathrm{~mL} / \mathrm{mmol})$. The layers were separated and the aqueous phase was extracted with $\mathrm{CH}_{2} \mathrm{Cl}_{2}$ (3 times, $4 \mathrm{~mL} / \mathrm{mmol}$ ). The combined organic layers were washed with a saturated solution of $\mathrm{NaCl}(1 \mathrm{~mL} / \mathrm{mmol})$, dried over $\mathrm{MgSO}_{4}$, filtered and concentrated under reduced pressure to give a crude product that was purified by chromatography on silica gel using the appropriate mixture of solvents.

\subsection{Synthesis of (-)-(2S,3R)-2-n-Butyl-3-(p-tolylsulfonyl)-3,6-dihydro-2H-pyran, 9a.}

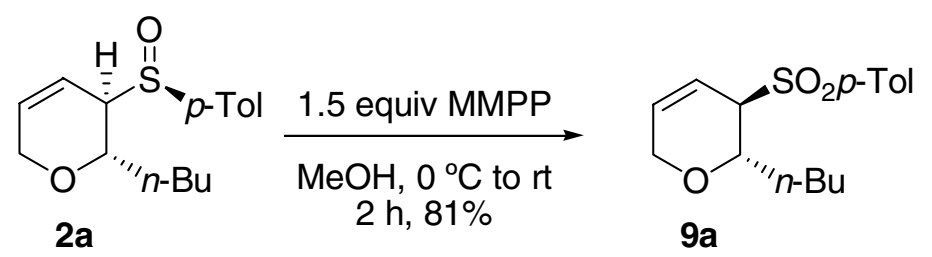

From sulfoxide 2a (7 mg, $0.026 \mathrm{mmol})$ and MMPP (24 mg (80\%), $0.039 \mathrm{mmol})$, following the general procedure $(3 \mathrm{~h})$ sulfone 9a was obtained. Purification by chromatography (10-30\% EtOAchexane) afforded $6 \mathrm{mg}(0.021 \mathrm{mmol}, 81 \%)$ of $9 \mathrm{a}$ as a colorless oil.

Data for 9a: $\boldsymbol{R}_{f}=0.38\left(30 \%\right.$ EtOAc-hexane). $[\alpha]^{20}{ }_{\mathbf{D}}=-150.6(c=0.59) .{ }^{1} \mathbf{H}$ NMR (300 MHz) $\delta 0.87(\mathrm{t}, 3 \mathrm{H}, J=7.1 \mathrm{~Hz}, \mathrm{Me}-n-\mathrm{Bu}), 1.20-1.40$ (m, $4 \mathrm{H}), 1.62$ (m, $2 \mathrm{H}), 2.42$ (s, $3 \mathrm{H}, \mathrm{Me}-p$-Tol), 3.51 (m, $1 \mathrm{H}, \mathrm{H}-3$ ), 3.63 (ddd, $1 \mathrm{H}, J=17.6,4.6,2.4 \mathrm{~Hz}, \mathrm{H}-6), 3.93$ (ddd, $1 \mathrm{H}, J=17.6,4.8,2.7 \mathrm{~Hz}, \mathrm{H}-6$ ), 4.13 (ddd, $1 \mathrm{H}, J=8.7,5.4,3.7 \mathrm{~Hz}, \mathrm{H}-2), 5.87$ (ddt, $1 \mathrm{H}, J=10.2,3.7,2.2 \mathrm{~Hz}), 6.00$ (dtd, $1 \mathrm{H}, J=$ 10.2, 2.7, $1.5 \mathrm{~Hz}), 7.30\left(\mathrm{~d}, 2 \mathrm{H}, J=8.1 \mathrm{~Hz}, p\right.$-Tol), $7.71\left(\mathrm{~d}, 2 \mathrm{H}, J=8.3 \mathrm{~Hz}, p\right.$-Tol). ${ }^{13} \mathbf{C ~ N M R}(\mathbf{M H z})$ 
$\delta \quad 14.0,21.7,22.4,27.6,32.0,60.8,64.6,70.8,117.0,129.3,129.6,133.0,134.1,144.6$. IR (film): 3022, 2949, 2928, 2859, 1597, 1493, 1456, 1380, 1309, 1301, 1284, 1216, 1182, 1132, 1084, 1019, $814,758,708 \mathrm{~cm}^{-1}$. MS (ES): $611[2 \mathrm{M}+\mathrm{Na}]^{+}, 317[\mathrm{M}+\mathrm{Na}]^{+}, 295[\mathrm{M}+1]^{+}(100 \%)$.

\subsection{Synthesis of (-)-(2R,3R)-2-n-Butyl-3-(p-tolylsulfonyl)-3,6-dihydro-2H-pyran, $10 a$.}
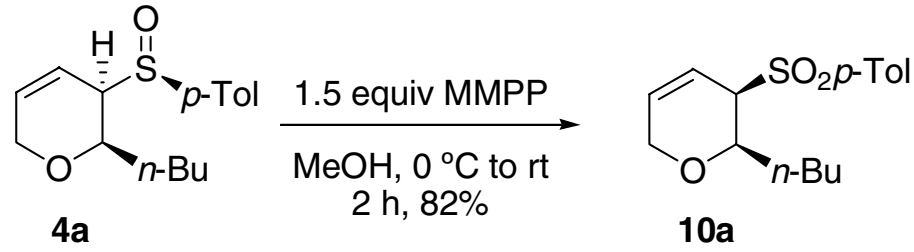

From sulfoxide 4a (6 mg, $0.022 \mathrm{mmol})$ and MMPP (20 mg (80\%), $0.033 \mathrm{mmol})$, following the general procedure $(3 \mathrm{~h})$ sulfone 10a was obtained. Purification by chromatography (10-30\% EtOAchexane) afforded $5 \mathrm{mg}(0.018 \mathrm{mmol}, 82 \%)$ of $\mathbf{1 0 a}$ as a colorless oil.

Data for 10a: $\quad \boldsymbol{R}_{f}=0.36\left(30 \%\right.$ EtOAc-hexane). $[\alpha]^{20}{ }_{\mathbf{D}}=-208.5(c=0.47) . \quad{ }^{1} \mathbf{H}$ NMR (300 MHz) $\delta 0.90(\mathrm{t}, 3 \mathrm{H}, J=7.1 \mathrm{~Hz}, \mathrm{Me}-n-\mathrm{Bu}), 1.31-1.50(\mathrm{~m}, 4 \mathrm{H}), 1.98(\mathrm{~m}, 2 \mathrm{H}), 2.41$ (s, $3 \mathrm{H}, \mathrm{Me}-p-$ Tol), 3.67 (m, 2 H, H-2, H-3), 3.78 (dm, 1 H, $J=17.1$ Hz, H-6), 4.03 (dm, 1 H, J = 17.1 Hz, H-6), 5.88 (ddt, $1 \mathrm{H}, J=10.2,4.9,1.9 \mathrm{~Hz}), 5.95(\mathrm{dm}, 1 \mathrm{H}, J=10.2 \mathrm{~Hz}), 7.27$ (d, $2 \mathrm{H}, J=8.1 \mathrm{~Hz}, p$-Tol), 7.72 (d, $2 \mathrm{H}, J=8.3 \mathrm{~Hz}, p$-Tol). ${ }^{13} \mathbf{C}$ NMR ( MHz) $\delta$ 14.0, 21.6, 22.5, 28.8, 29.7, 32.2, 63.7, 66.0, 75.1, 120.0, 129.1, 129.6, 132.9, 136.2, 144.2. IR (film): 3043, 3014, 2925, 2855, 2826, 1596, 1466, 1450, $1385,1371,1313,1286,1182,1136,1083,1035,1013,962,854,826,752,712 \mathrm{~cm}^{-1}$. MS (ES): 611 $[2 \mathrm{M}+\mathrm{Na}]^{+}(100 \%), 317[\mathrm{M}+\mathrm{Na}]^{+}, 295[\mathrm{M}+1]^{+}$.

\subsection{Synthesis of (-)-(2S,3R)-2-Phenyl-3-(p-tolylsulfonyl)-3,6-dihydro-2H-pyran, $9 \mathrm{~b}$.}

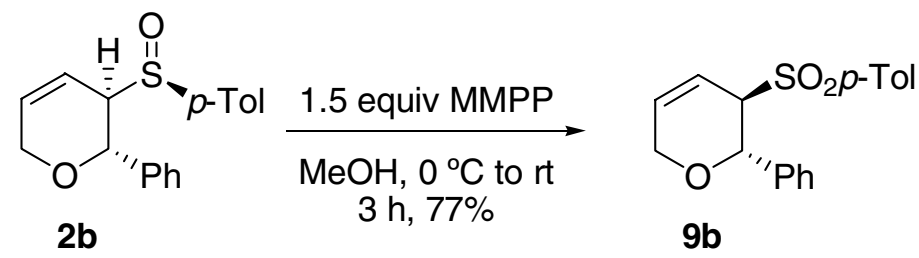

From sulfoxide 2b (10 mg, $0.033 \mathrm{mmol})$ and MMPP (31 mg (80\%), $0.049 \mathrm{mmol})$, following the general procedure $(3 \mathrm{~h})$ sulfone $9 \mathrm{~b}$ was obtained. Purification by chromatography (10-30\% EtOAchexane) afforded $8 \mathrm{mg}(0.025 \mathrm{mmol}, 77 \%)$ of $\mathbf{9 b}$ as a white solid that was recrystallized from $\mathrm{Et}_{2} \mathrm{O}$ hexane.

Data for 9b: $\mathbf{m p}: 173-175{ }^{\circ} \mathrm{C} . \quad \boldsymbol{R}_{f}=0.47(50 \%$ EtOAc-hexane $) .[\alpha]^{20}{ }_{\mathrm{D}}=-205.1(c=0.67) .{ }^{1} \mathbf{H}$ NMR (300 MHz) $\delta 2.39$ (s, $3 \mathrm{H}, \mathrm{Me}-p$-Tol), 3.83 (ap q, $2 \mathrm{H}, J=2.3 \mathrm{~Hz}, \mathrm{H}-6), 4.17$ (m, $1 \mathrm{H}, \mathrm{H}-3)$, 
$5.20(\mathrm{~d}, 1 \mathrm{H}, J=4.1 \mathrm{~Hz}, \mathrm{H}-2), 6.08$ (dtd, $1 \mathrm{H}, J=10.5,2.4,1.5 \mathrm{~Hz}, \mathrm{H}-5), 6.16$ (dquint, $1 \mathrm{H}, J=10.2$, $1.9 \mathrm{~Hz}, \mathrm{H}-4), 7.19-7.27(\mathrm{~m}, 7 \mathrm{H}), 7.66\left(\mathrm{~d}, 2 \mathrm{H}, J=8.3 \mathrm{~Hz}, p\right.$-Tol). ${ }^{13} \mathbf{C}$ NMR (50 MHz) $\delta 21.6(\mathrm{Me}-p-$ Tol), 61.8, 63.4, 73.2, 117.2, 127.6 (2 C), 128.5 (2 C), 129.2 (2 C), 129.3 (2 C), 133.6, 134.4, 137.4, 144.5. IR (KBr): 3028, 2898, 2833, 1631, 1595, 1299, 1134, 1084, 810, 748, $708 \mathrm{~cm}^{-1}$. MS (ES): 651 $[2 \mathrm{M}+\mathrm{Na}]^{+}(100 \%), 337[\mathrm{M}+\mathrm{Na}]^{+}, 315[\mathrm{M}+1]^{+}$. Anal. calcd for $\mathrm{C}_{18} \mathrm{H}_{18} \mathrm{O}_{3} \mathrm{~S}: \mathrm{C}, 68.76 ; \mathrm{H}, 5.77 ; \mathrm{S}, 10.20$.

Found: C, 68.98; H, 5.59; S, 10.47.

\subsection{Synthesis of (-)-(2R,3S)-2-Phenyl-3-(p-tolylsulfonyl)-3,6-dihydro-2H-pyran, ent-9b.}

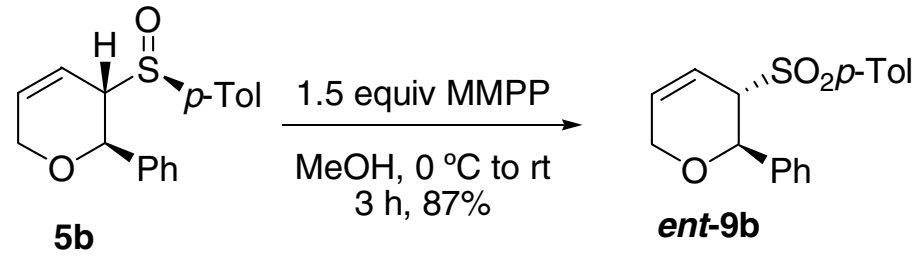

From sulfoxide 5b (10 mg, $0.033 \mathrm{mmol})$ and MMPP (31 mg (80\%), $0.049 \mathrm{mmol})$, following the general procedure $(3 \mathrm{~h})$ sulfone ent-9b was obtained. Purification by chromatography (10-30\% EtOAc-hexane) afforded $9 \mathrm{mg}(0.028 \mathrm{mmol}, 87 \%)$ of $\boldsymbol{e n t - 9 b}$ as a white solid that was recrystallized from $\mathrm{Et}_{2} \mathrm{O}$-hexane.

Data for $\boldsymbol{e n t - 9 b}$ was identical to that described above for $9 \mathrm{~b}$ except for optical rotation: $[\alpha]^{20}{ }_{\mathrm{D}}=$ $+196.4(c=0.83)$.

\subsection{Synthesis of (-)-(2S,3R,6R)-6-Methyl-2-phenyl-3-(p-tolylsulfonyl)-3,6-dihydro-2H-pyran, 9 d.}

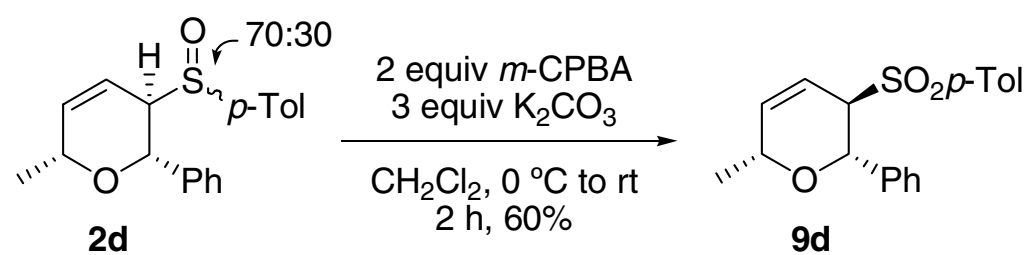

From a 70:30 mixture of sulfoxides $\left(\boldsymbol{R}_{S}\right):\left(\boldsymbol{S}_{S}\right)-\mathbf{2 d}(3 \mathrm{mg}, 0.010 \mathrm{mmol}), \mathrm{K}_{2} \mathrm{CO}_{3}(4 \mathrm{mg}, 0.030$ mmol) and $m$-CPBA (4 mg (70\%), $0.015 \mathrm{mmol})$, following the general procedure $(2 \mathrm{~h})$, a single sulfone 9d was obtained. Purification by chromatography afforded $2 \mathrm{mg}(0.006 \mathrm{mmol}, 60 \%)$ of 9d as a colorless oil.

Data for 9d: $\boldsymbol{R}_{f}=0.38\left(30 \%\right.$ EtOAc-hexane). $[\alpha]^{20}{ }_{\mathbf{D}}=-53.0(c=0.16) .{ }^{1} \mathbf{H}$ NMR (400 MHz) $\delta 1.20(\mathrm{~d}, 3 \mathrm{H}, J=6.8 \mathrm{~Hz}, \mathrm{Me}-6), 2.34$ (s, $3 \mathrm{H}, \mathrm{Me}-p$-Tol), 4.25 (m, 2 H, H-3, H-6), 6.09 (d, $1 \mathrm{H}, J=$ 11.2 Hz ), $6.13(\mathrm{~d}, 1 \mathrm{H}, J=11.2 \mathrm{~Hz}), 7.09(\mathrm{~d}, 2 \mathrm{H}, J=8.1 \mathrm{~Hz}), 7.17-7.22(\mathrm{~m}, 5 \mathrm{H}), 7.45(\mathrm{~d}, 2 \mathrm{H}, J=$ $8.2 \mathrm{~Hz}) .{ }^{13} \mathrm{C}$ NMR (100 MHz, partial data) $\delta 20.8(\mathrm{Me}-6), 21.5(\mathrm{Me}-p$-Tol), 65.2, 71.1, $118.1(\mathrm{C}-4)$, 
128.0 (2 C), 128.4 (2 C), 128.7, 129.3 (2 C), 137.9 (C-5). IR (film): 3021, 2926, 2855, 1595, 1494, $1456,1374,1313,1303,1215,1144,1085,1042,758 \mathrm{~cm}^{-1}$. MS (ES): 679 [2M+Na] $]^{+}(100 \%), 351$ $[\mathrm{M}+\mathrm{Na}]^{+}, 329[\mathrm{M}+1]^{+}$.

\subsection{Synthesis of (2S,3R)-4-Methyl-2-phenyl-3-(p-tolylsulfonyl)-3,6-dihydro-2H-pyran, 9e.}

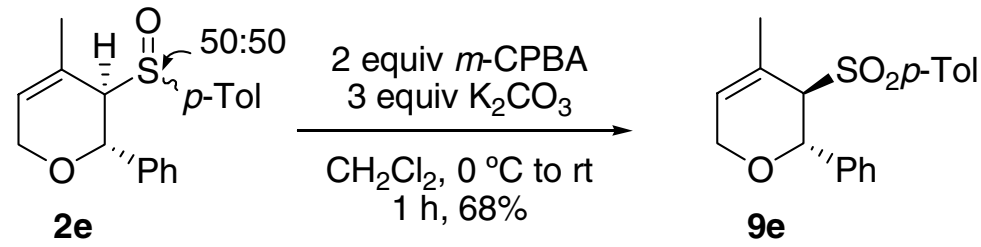

From a 50:50 mixture of sulfoxides $\left(\boldsymbol{R}_{s}\right):\left(S_{S}\right)-2 \mathrm{e}(7 \mathrm{mg}, 0.022 \mathrm{mmol}), \mathrm{K}_{2} \mathrm{CO}_{3}(9 \mathrm{mg}, 0.066$ $\mathrm{mmol})$ and $\mathrm{m}$-CPBA $(8 \mathrm{mg}(70 \%), 0.034 \mathrm{mmol})$, following the general procedure $(1 \mathrm{~h})$, a single sulfone 9e was obtained. Purification by chromatography afforded $5 \mathrm{mg}(0.015 \mathrm{mmol}, 68 \%)$ of $9 \mathrm{e}$ as a white solid that was recrystallized from EtOAc-hexane.

Data for 9e: $\mathbf{m p}: 107-109{ }^{\circ} \mathrm{C} . \quad \boldsymbol{R}_{f}=0.42\left(30 \%\right.$ EtOAc-hexane). $[\alpha]^{20}{ }_{\mathbf{b}}=-233.3(c=0.45) .{ }^{1} \mathbf{H}$ NMR (300 MHz) $\delta 2.12(\mathrm{~d}, 3 \mathrm{H}, J=1.7 \mathrm{~Hz}, \mathrm{Me}-4), 2.42(\mathrm{~s}, 3 \mathrm{H}, \mathrm{Me}-p$-Tol), 3.34 (dm, $1 \mathrm{H}, J=17.6$ Hz, H-6), 3.56 (dd, 1 H, J = 17.6, 1.7 Hz, H-6), 3.99 (s, 1 H, H-3), 5.48 (br s, 1 H, H-2), 5.66 (s, 1 H, H-5), 7.19-7.22 (m, $2 \mathrm{H}), 7.26-7.32(\mathrm{~m}, 5 \mathrm{H}), 7.81$ (d, $2 \mathrm{H}, J=8.3 \mathrm{~Hz}, p$-Tol). ${ }^{13} \mathbf{C}$ NMR (75 MHz) $\delta$ 21.7, 23.3, 60.2, 67.7, 71.7, 124.1, 126.9 (2 C), 128.1, 128.5 (3 C), 129.0 (2 C), 129.8 (2 C), 134.6, 137.3, 144.4. IR (KBr): 3028, 2920, 2826, 1630, 1285, 1131, 1084, $694 \mathrm{~cm}^{-1}$. MS (ES): 679 $[2 \mathrm{M}+\mathrm{Na}]^{+}(100 \%), 351[\mathrm{M}+\mathrm{Na}]^{+}, 329[\mathrm{M}+1]^{+}$. Anal. calcd for $\mathrm{C}_{19} \mathrm{H}_{20} \mathrm{O}_{3} \mathrm{~S}: \mathrm{C}, 69.48 ; \mathrm{H}, 6.14 ; \mathrm{S}, 9.76$. Found: C, 69.25; H, 6.43; S, 9.52.

\section{General Procedure for Osmium-Catalyzed Dihydroxylation-Oxidation of 3-Sulfinyl-dihydro-} $2 H$-pyrans.

To a solution of the corresponding sulfoxide in a 9:1 mixture of acetone and $\mathrm{H}_{2} \mathrm{O}(0.1 \mathrm{M})$, at $\mathrm{rt}$, 4 equiv of $\mathrm{Me}_{3} \mathrm{NO}$ and 0.05 equiv of $\mathrm{OsO}_{4}$ were added. The reaction was stirred until completion and then quenched with a solution of aqueous $\mathrm{Na}_{2} \mathrm{~S}_{2} \mathrm{O}_{4}(1 \mathrm{M}, 5 \mathrm{~mL} / \mathrm{mmol})$. The solvent was evaporated and the crude product was filtered through a short pad of silica gel.

\subsection{Synthesis of (+)-(3S,4S,5S,6S)-4-Methyl-6-phenyl-5-(p-tolylsulfonyl)-tetrahydro-2H-pyran-} 3,4-diol, 11. 
S15

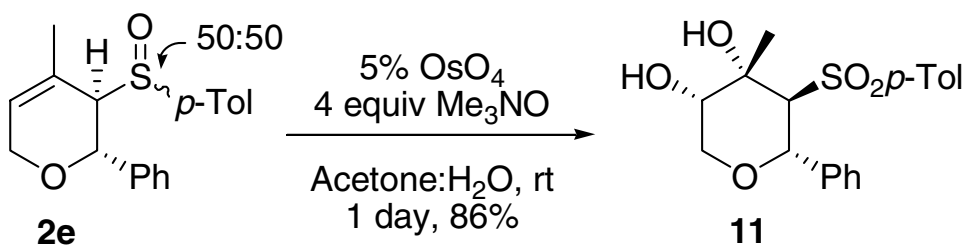

From sulfoxide $2 \mathrm{e}(9 \mathrm{mg}, 0.029 \mathrm{mmol}), \mathrm{Me}_{3} \mathrm{NO}(13 \mathrm{mg}, 0.116 \mathrm{mmol})$ and $\mathrm{OsO}_{4}(25 \mu \mathrm{L}(2.5 \%$ wt), $0.002 \mathrm{mmol}$ ), following the general procedure (1 day), diol 11 was obtained. Purification by chromatography (50\% EtOAc-hexane) afforded $9 \mathrm{mg}(0.025 \mathrm{mmol}, 86 \%)$ of $\mathbf{1 1}$ as a white solid that was recrystallized from EtOAc-hexane.

Data for 11: mp: $188-190{ }^{\circ} \mathrm{C} . \quad \boldsymbol{R}_{f}=0.10(80 \%$ EtOAc $) .[\alpha]^{20}{ }_{\mathbf{D}}=+45.4(c=0.32) .{ }^{1} \mathbf{H}$ NMR (300 MHz) $\delta 1.89$ (s, 3 H, Me-4), 2.28 (s, 3 H, Me-p-Tol), 3.27 (m, 1 H, H-3), 3.60 (s, 1 H, OH), 3.85 (ddd, $1 \mathrm{H}, J=12.9,2.3,1.3 \mathrm{~Hz}, \mathrm{H}-2), 4.05$ (dd, $1 \mathrm{H}, J=12.9,1.9 \mathrm{~Hz}, \mathrm{H}-2), 4.13$ (d, $2 \mathrm{H}, J=10.2 \mathrm{~Hz}$, H-5), 4.58 (d, 2 H, $J=10.2 \mathrm{~Hz}, \mathrm{H}-6), 6.88$ (d, $2 \mathrm{H}, J=8.1 \mathrm{~Hz}$ ), 6.93-7.00 (m, $4 \mathrm{H}), 7.04-7.10$ (m, $3 \mathrm{H})$. ${ }^{13}$ C NMR (50 MHz) $\delta$ 21.4, 21.8, 67.5, 70.0, 72.9, 74.1, 78.0, 126.9 (2 C), 128.0 (2 C), 128.7, 128.9 (2 C), 129.1 (2 C), 136.3, 137.6, 143.5. IR (KBr): 3513, 3434, 2920, 1285, 1133, 1017, $717 \mathrm{~cm}^{-1}$. MS (ES): $747[2 \mathrm{M}+\mathrm{Na}]^{+}(100 \%), 385[\mathrm{M}+\mathrm{Na}]^{+}$. Anal. calcd for $\mathrm{C}_{19} \mathrm{H}_{22} \mathrm{O}_{5} \mathrm{~S}: \mathrm{C}, 62.96 ; \mathrm{H}, 6.12 ; \mathrm{S}, 8.85$.

Found: C, 62.78; H, 6.42; S, 8.64.

\subsection{Synthesis of (+)-(3S,4S,5R,6S)-6-Phenyl-5-(p-tolylsulfonyl)-tetrahydro-2H-pyran-3,4-diol, 12.}

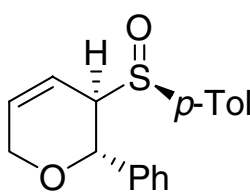

2b

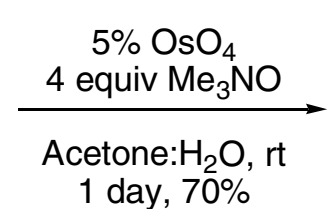

1 day, $70 \%$

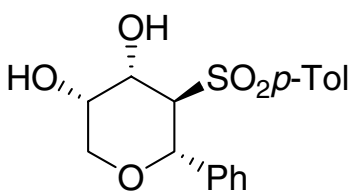

12

From sulfoxide $2 \mathbf{b}(90 \mathrm{mg}, 0.300 \mathrm{mmol}), \mathrm{Me}_{3} \mathrm{NO}(133 \mathrm{mg}, 1.200 \mathrm{mmol})$ and $\mathrm{OsO}_{4}(0.19 \mathrm{~mL}$ ( $2.5 \% \mathrm{wt}), 0.015 \mathrm{mmol})$, following the general procedure (1 day), diol 12 was obtained. Purification by chromatography (30-80\% EtOAc-hexane) afforded $74 \mathrm{mg}(0.21 \mathrm{mmol}, 70 \%)$ of $\mathbf{1 2}$ as a white solid that was recrystallized from EtOAc-hexane.

Data for 12: $\mathbf{m p}: 133-135^{\circ} \mathrm{C} . \quad \boldsymbol{R}_{f}=0.32\left(80 \%\right.$ EtOAc-hexane). $[\alpha]^{20}{ }_{\mathbf{D}}=+35.0(c=0.48) .{ }^{1} \mathbf{H}$ NMR (300 MHz)-COSY $\delta 2.29$ (s, $3 \mathrm{H}, \mathrm{Me}-p$-Tol), $2.88(\mathrm{~s}, 1 \mathrm{H}, \mathrm{OH}), 3.71(\mathrm{dm}, 1 \mathrm{H}, J=12.7 \mathrm{~Hz}$, H-2), 3.93 (t, $1 \mathrm{H}, J=10.1 \mathrm{~Hz}, \mathrm{H}-5), 4.08$ (m, $1 \mathrm{H}, \mathrm{H}-3$ ), 4.14 (dd, 1 H, $J=12.7,2.2 \mathrm{~Hz}, \mathrm{H}-2), 4.45$ (d, $1 \mathrm{H}, J=10.0 \mathrm{~Hz}, \mathrm{H}-6$ ), 4.57 (ddd, $1 \mathrm{H}, J=10.2,3.4,1.6 \mathrm{~Hz}, \mathrm{H}-4$ ), 5.15 (dd, $1 \mathrm{H}, J=1.5,0.5 \mathrm{~Hz}, \mathrm{OH}$ ), $6.92(\mathrm{~d}, 2 \mathrm{H}, J=8.5 \mathrm{~Hz}), 6.98-7.12(\mathrm{~m}, 7 \mathrm{H}) .{ }^{13} \mathrm{C}$ NMR (75 MHz)-HSQC $\delta 21.5$ (Me-p-Tol), 67.3 (C-3), 67.6 (C-5), 67.9 (C-4), 69.5 (C-6), 78.7 (C-2), 127.4 (2 C), 128.2 (2 C), 128.8 (2 C), 129.2 (2 C), 136.1, 136.2, 144.1. IR (KBr): 3503, 3065, 2913, 2876, 1627, 1595, 1288, 1230, 1138, 1097, 882, 
813, 768, $724 \mathrm{~cm}^{-1}$. MS (ES): $719[2 \mathrm{M}+\mathrm{Na}]^{+}(100 \%), 371[\mathrm{M}+\mathrm{Na}]^{+}, 349[\mathrm{M}+1]^{+}$. Anal. calcd for $\mathrm{C}_{18} \mathrm{H}_{20} \mathrm{O}_{5} \mathrm{~S}: \mathrm{C}, 62.05 ; \mathrm{H}, 5.79 ; \mathrm{S}, 9.20$. Found: C, 62.36; H, 5.47; S, 9.31.

\section{Synthesis of (-)-(3R,6S)-6-Phenyl-5-(p-tolylsulfonyl)-3,6-dihydro-2H-pyran-3-ol, $13 .{ }^{2}$}

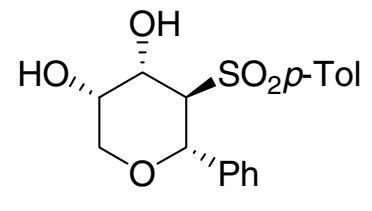

12

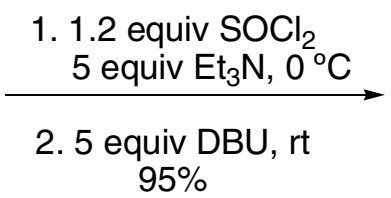

$95 \%$<smiles>O=S(=O)(O[Na])C1=C[C@H](O)CO[C@H]1P</smiles>

13

To a cold $\left(0^{\circ} \mathrm{C}\right)$ solution of diol $12(70 \mathrm{mg}, 0.20 \mathrm{mmol})$ in $\mathrm{CH}_{2} \mathrm{Cl}_{2}$, under an argon atmosphere, was added $\mathrm{Et}_{3} \mathrm{~N}\left(0.14 \mathrm{~mL}, 1.00 \mathrm{mmol}, 5.0\right.$ equiv) followed by $\mathrm{SOCl}_{2}(18 \mu \mathrm{L}, 0.24 \mathrm{mmol}, 1.2$ equiv). The reaction mixture was stirred for $30 \mathrm{~min}$ at $0{ }^{\circ} \mathrm{C}$ and then concentrated under reduced pressure for $15 \mathrm{~min}$. The residue was redissolved in $\mathrm{CH}_{2} \mathrm{Cl}_{2}(2 \mathrm{~mL})$ under argon, and DBU $(0.15 \mathrm{~mL}, 1.00 \mathrm{mmol}$, 5.0 equiv) was added. The mixture was stirred at $\mathrm{rt}$ until completion (30 min) and quenched with a saturated solution of $\mathrm{NH}_{4} \mathrm{Cl}(2 \mathrm{~mL} / \mathrm{mmol})$ and water $(2 \mathrm{~mL} / \mathrm{mmol})$. The layers were separated and the aqueous phase was extracted twice with $\mathrm{CH}_{2} \mathrm{Cl}_{2}$. The combined organic extracts were washed with a saturated solution of $\mathrm{NaCl}$, dried over anhydrous $\mathrm{MgSO}_{4}$ and concentrated. Purification by chromatography (30-80\% EtOAc-hexane) afforded $63 \mathrm{mg}(0.19 \mathrm{mmol}, 95 \%)$ of 13 as a white solid that was recrystallized from EtOAc-hexane.

Data for 13: $\mathbf{m p}$ : $126-128{ }^{\circ} \mathrm{C} . \quad \boldsymbol{R}_{f}=0.32\left(50 \%\right.$ EtOAc-hexane). $[\alpha]^{20}{ }_{\mathbf{D}}=-37.0(c=0.50) .{ }^{1} \mathbf{H}$ NMR (300 MHz)-COSY $\delta 2.06(\mathrm{~d}, 1 \mathrm{H}, J=8.8 \mathrm{~Hz}, \mathrm{OH}), 2.31$ (s, $3 \mathrm{H}, \mathrm{Me}-p$-Tol), 3.75 (dd, $1 \mathrm{H}, J=$ 12.0, 3.5 Hz, H-2), 3.84 (dd, $1 \mathrm{H}, J=12.0,3.5 \mathrm{~Hz}, \mathrm{H}-2), 4.38$ (m, $1 \mathrm{H}, \mathrm{H}-3), 5.40$ (t, $1 \mathrm{H}, J=1.6 \mathrm{~Hz}$, H-6), 6.96 (d, 2 H, $J=7.8 \mathrm{~Hz}), 7.01-7.04(\mathrm{~m}, 5 \mathrm{H}), 7.14$ (d, $2 \mathrm{H}, J=8.3 \mathrm{~Hz}), 7.37$ (ddd, $1 \mathrm{H}, J=4.9$, 1.7, $0.5 \mathrm{~Hz}, \mathrm{H}-4)$. NOESY-1D between H-2 (3.84 ppm)/H-3: 3.4\%; between H-2 (3.75 ppm)/H-6: 5.4\%; between H-2/H-3 (3.75 ppm): 6.2\%; between $\mathrm{H}-3 / \mathrm{H}-2$ (3.84 ppm): 1.9\%; between $\mathrm{H}-3 / \mathrm{H}-2$ (3.75 ppm): 2.4\%; between H-3/H-4: 4.6\%; between H-4/H-3: 4.5\%; between H-6/H-2: $2.7 \%$. ${ }^{13} \mathbf{C}$ NMR (75 MHz) $\delta 21.5$ (Me-p-Tol), 62.5 (C-2), 67.2, 76.2, 127.7 (2 C), 128.1 (2 C), 128.7, 129.2 (2 C), 129.3 (2 C), 135.4, 136.3, 137.7, 143.8, 145.4. IR (KBr): 3484, 2971, 2913, 2855, 1631, 1302, 1142, 1086, 1056, 829, 761, $702 \mathrm{~cm}^{-1}$. MS (ES): $683[2 \mathrm{M}+\mathrm{Na}]^{+}, 353[\mathrm{M}+\mathrm{Na}]^{+}(100 \%), 331[\mathrm{M}+1]^{+}$. Anal. calcd for $\mathrm{C}_{18} \mathrm{H}_{18} \mathrm{O}_{4} \mathrm{~S}$ : C, 65.43; H, 5.49; S, 9.70. Found: C, 65.67; H, 5.24; S, 9.56.

\section{Synthesis of $(3 R, 6 S)$-6-Phenyl-5-(p-tolylsulfonyl)-3,6-dihydro-2H-pyran-3-yl $N$-benzyl carbamate, 14.}




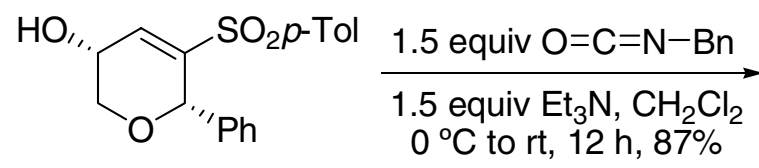

13

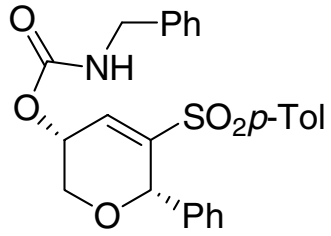

14

To a solution of sulfone $13(10 \mathrm{mg}, 0.030 \mathrm{mmol})$ in $\mathrm{CH}_{2} \mathrm{Cl}_{2}$ at $0^{\circ} \mathrm{C}$, under an argon atmosphere, was added of $\mathrm{Et}_{3} \mathrm{~N}(5 \mu \mathrm{L}, 0.036 \mathrm{mmol}, 1.5$ equiv) and of benzyl isocyanate (6 $\mu \mathrm{L}, 0.045 \mathrm{mmol}, 1.5$ equiv). The mixture was stirred overnight and then water $(3 \mathrm{~mL} / \mathrm{mmol})$ was added. The layers were separated and the aqueous phase was extracted with $\mathrm{CH}_{2} \mathrm{Cl}_{2}(5 \mathrm{~mL} / \mathrm{mmol}, 3$ times $)$. The combined organic extracts were washed with a saturated solution of $\mathrm{NaCl}$, dried over $\mathrm{MgSO}_{4}$, filtered and concentrated. Purification by chromatography (1-5\% EtOAc- $\left.\mathrm{CH}_{2} \mathrm{Cl}_{2}\right)$ afforded $13 \mathrm{mg}(0.028 \mathrm{mmol}$, 93\%) of $\mathbf{1 4}$ as a white solid that was recrystallized from EtOAc-hexane.

Data for 14: $\mathbf{m p}: 52-54{ }^{\circ} \mathrm{C} . \quad \boldsymbol{R}_{f}=0.44\left(50 \%\right.$ EtOAc-hexane). $[\alpha]^{20}{ }_{\mathbf{D}}=-85.1(c=0.41) .{ }^{1} \mathbf{H}$ NMR (300 MHz)-COSY $\delta 2.31$ (s, 3 H, Me-p-Tol), 3.79 (dd, 1 H, J = 12.6, 4.3 Hz, H-Bn), 3.89 (dd, $1 \mathrm{H}, J=12.5,3.9 \mathrm{~Hz}, \mathrm{H}-\mathrm{Bn}), 4.38$ (m, $1 \mathrm{H}, \mathrm{H}-2), 5.10$ (br t, $1 \mathrm{H}, \mathrm{NH}), 5.38$ (s, $1 \mathrm{H}, \mathrm{H}-6), 5.39$ (m, $1 \mathrm{H}$, H-3), 6.98 (dm, $2 \mathrm{H}, J=7.8 \mathrm{~Hz}), 7.02-7.07(\mathrm{~m}, 4 \mathrm{H}), 7.12-7.20(\mathrm{~m}, 3 \mathrm{H}), 7.27-7.36(\mathrm{~m}, 5 \mathrm{H}) .{ }^{13} \mathbf{C}$ NMR (50 MHz) $\delta$ 21.5, 45.3, 64.3, 64.4, 75.6, 127.6, 127.8 (2 C), 128.1 (2 C), 128.8 (4 C), 129.3 (4 C), 134.5, 136.2, 137.9, 143.9, 147.2, 155.3. IR (film): 3426, 3057, 3028, 2920, 1723, 1318, 1148, 1091, $700 \mathrm{~cm}^{-1}$. MS (ES): $949[2 \mathrm{M}+\mathrm{Na}]^{+}, 486[\mathrm{M}+\mathrm{Na}]^{+}(100 \%), 464[\mathrm{M}+1]^{+}$. Anal. calcd for $\mathrm{C}_{26} \mathrm{H}_{25} \mathrm{NO}_{5} \mathrm{~S}: \mathrm{C}, 67.37 ; \mathrm{H}, 5.44 ; \mathrm{N}, 3.02 ; \mathrm{S}, 6.92$. Found: C, 67.56; H, 5.13; N, 3.14; S, 6.64 .

\section{Synthesis of (+)-(3aR,6S,7R,7aS)-1-Benzyl-6-phenyl-7-(p-tolylsulfonyl)-tetrahydro-1H-} pyrano[4,3-d]oxazol-2(6H)-one, 15.

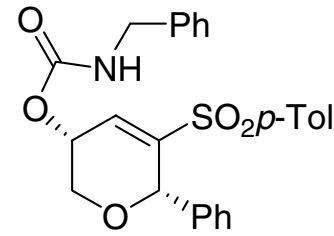

14

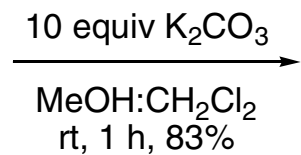

rt, $1 \mathrm{~h}, 83 \%$

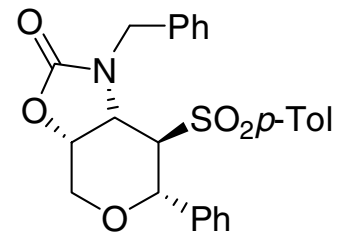

15

To a solution of sulfone $14(6 \mathrm{mg}, 0.013 \mathrm{mmol})$ in a $1: 1$ mixture of $\mathrm{MeOH}$ and $\mathrm{H}_{2} \mathrm{O}(0.1 \mathrm{M})$ at rt, was added $\mathrm{K}_{2} \mathrm{CO}_{3}(18 \mathrm{mg}, 0.13 \mathrm{mmol}, 10$ equiv). The mixture was stirred at $\mathrm{rt}$ and monitored by TLC until completion $(1 \mathrm{~h})$. Then water $(3 \mathrm{~mL} / \mathrm{mmol})$ was added, $\mathrm{MeOH}$ was removed under reduced pressure and the residue was diluted with EtOAc $(5 \mathrm{~mL} / \mathrm{mmol})$. The layers were separated and the aqueous phase was extracted with EtOAc $(5 \mathrm{~mL} / \mathrm{mmol}, 3$ times $)$. The combined organic extracts were washed with a saturated solution of $\mathrm{NaCl}$, dried over $\mathrm{MgSO}_{4}$, filtered and concentrated. Purification by 
chromatography (20-50\% EtOAc-hexane) afforded $5 \mathrm{mg}(0.011 \mathrm{mmol}, 85 \%)$ of $\mathbf{1 5}$ as a white solid that was recrystallized from EtOAc-hexane.

Data for 15: $\mathbf{m p}: 223-225^{\circ} \mathrm{C} . \quad \boldsymbol{R}_{f}=0.45(50 \%$ EtOAc-hexane $) . \quad[\alpha]^{20}{ }_{\mathbf{D}}=+5.9(c=0.55) .{ }^{1} \mathbf{H}$ NMR (300 MHz)-COSY $\delta 2.46$ (s, 3 H, Me-p-Tol), 3.15 (d, 1 H, J = 15.6 Hz, H-Bn), 3.48 (dd, 1 H, $J=3.7,1.5 \mathrm{~Hz}, \mathrm{H}-7), 4.15$ (dd, $1 \mathrm{H}, J=13.7,1.9 \mathrm{~Hz}, \mathrm{H}-4), 4.21$ (d, $1 \mathrm{H}, J=13.4 \mathrm{~Hz}, \mathrm{H}-4), 4.22$ (dd, 1 $\mathrm{H}, J=9.8,1.2 \mathrm{~Hz}, \mathrm{H}-7 \mathrm{a}), 4.63$ (d, $1 \mathrm{H}, J=15.6 \mathrm{~Hz}, \mathrm{H}-\mathrm{Bn}), 4.75$ (dd, $1 \mathrm{H}, J=9.5,1.2 \mathrm{~Hz}, \mathrm{H}-3 \mathrm{a}), 5.49$ (d, $1 \mathrm{H}, J=3.7 \mathrm{~Hz}, \mathrm{H}-6), 6.69$ (d, $2 \mathrm{H}, J=7.1 \mathrm{~Hz}), 7.02(\mathrm{t}, 2 \mathrm{H}, J=7.4 \mathrm{~Hz}), 7.13$ (tm, $1 \mathrm{H}, J=7.3$ Hz), 7.21-7.36 (m, 7 H), 7.54 (d, $2 \mathrm{H}, J=8.3 \mathrm{~Hz})$. NOESY-1D between H-7/H-6: 2.5\%; between H7/H-7a: 4.1\%; between H-7/Bn: 5.8\%. ${ }^{13} \mathbf{C}$ NMR (50 MHz)-HSQC $\delta 21.8$ (Me- $p$-Tol), $45.7\left(\mathrm{CH}_{2}-\right.$ Bn), 49.4 (C-7a), 62.5 (C-3a), 67.5 (C-4), 69.6 (C-7), 73.7 (C-6), 125.3 (2 C), 127.8, 127.9 (2 C), 128.2, 128.6 (2 C), 128.7 (2 C), 129.0 (2 C), 130.3 (2 C), 133.8, 134.0, 141.5, 145.8, 157.5. IR (KBr): 3461, 3028, 2920, 1734, 1446, 1287, 1142, $699 \mathrm{~cm}^{-1}$. MS (ES): 949 [2M+Na] $486[\mathrm{M}+\mathrm{Na}]^{+}, 464$ $[\mathrm{M}+1]^{+}(100 \%)$. Anal. calcd for $\mathrm{C}_{26} \mathrm{H}_{25} \mathrm{NO}_{5} \mathrm{~S}: \mathrm{C}, 67.37 ; \mathrm{H}, 5.44 ; \mathrm{N}, 3.02 ; \mathrm{S}, 6.92$. Found: C, 67.26; H, $5.52 ; \mathrm{N}, 3.26 ; \mathrm{S}, 6.98$.

10. Synthesis of (-)-(2S,3R,4R,5S)-2-Phenyl-3-(p-tolylsulfonyl)-3,4-epoxy-tetrahydro-2H-pyran-3ol, 16.<smiles>[O+]O[Se]C1=C[C@H](O)CO[C@H]1c1ccccc1</smiles>

13

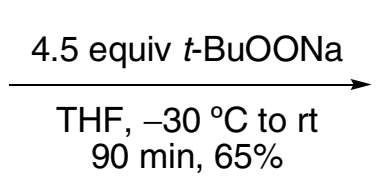

$90 \mathrm{~min}, 65 \%$

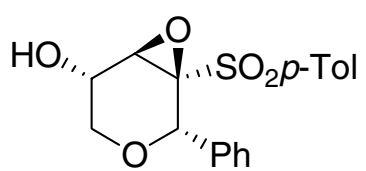

16

A two-necked round-bottomed flask fitted with a tube in T for entrance and exit of argon and a polyethylene stopper, was charged with anhydrous THF $(10 \mathrm{~mL} / \mathrm{mmol})$ and 4.0 equiv of oil free $\mathrm{NaH}$ (9 mg, $0.36 \mathrm{mmol})$. The mixture was cooled to $0{ }^{\circ} \mathrm{C}$ and then 4.5 equiv of $t$-BuOOH $(51 \mu \mathrm{L}, 0.40$ mmol, $80 \%$ in $t$-BuOO- $t$-Bu) was added. After stirring at $\mathrm{rt}$ for $30 \mathrm{~min}$, the resulting mixture was cooled to $-30{ }^{\circ} \mathrm{C}$ and a solution of sulfone $13(30 \mathrm{mg}, 0.09 \mathrm{mmol})$ in THF (10 mL/mmol), was added dropwise. The reaction mixture was allowed to warm to $\mathrm{rt}$ until completion $(1 \mathrm{~h} 30 \mathrm{~min})$ then quenched with a $1 \mathrm{M}$ solution of $\mathrm{Na}_{2} \mathrm{~S}_{2} \mathrm{O}_{4}(4 \mathrm{~mL} / \mathrm{mmol})$, diluted whith EtOAc and the layers were separated. The aqueous phase was extracted with EtOAc (3 times, $10 \mathrm{~mL} / \mathrm{mmol}$ ) and the combined organic extracts were washed with a saturated solution of $\mathrm{NaCl}(4 \mathrm{~mL} / \mathrm{mmol})$, dried over anhydrous $\mathrm{MgSO}_{4}$, filtered and concentrated under reduced pressure. Purification by chromatography (30-50\% EtOAc-hexane) afforded $20 \mathrm{mg}(0.058 \mathrm{mmol}, 65 \%)$ of $\mathbf{1 6}$ as a white solid that was recrystallized from $\mathrm{Et}_{2} \mathrm{O}$-hexane. 
Data for 16: $\mathbf{m p}: 58-60{ }^{\circ} \mathrm{C} . \quad \boldsymbol{R}_{f}=0.30(50 \%$ EtOAc-hexane $) . \quad[\alpha]^{20}{ }_{\mathbf{D}}=-90.0(c=0.10) .{ }^{1} \mathbf{H}$ NMR (300 MHz) $\delta 2.28(\mathrm{~s}, 3 \mathrm{H}, \mathrm{Me}-p$-Tol), $2.54(\mathrm{~d}, 1 \mathrm{H}, J=11.7 \mathrm{~Hz}, \mathrm{OH}), 3.62(\mathrm{dd}, 1 \mathrm{H}, J=12.7$, $2.93 \mathrm{~Hz}, \mathrm{H}-6), 3.89$ (dt, $1 \mathrm{H}, J=12.7,1.2 \mathrm{~Hz}, \mathrm{H}-6), 4.13$ (m, $1 \mathrm{H}, \mathrm{H}-5), 4.42$ (dd, $1 \mathrm{H}, J=5.1,1.0$ Hz, H-4), 5.21 (s, $1 \mathrm{H}, \mathrm{H}-2), 6.91(\mathrm{~d}, 2 \mathrm{H}, J=8.5 \mathrm{~Hz}), 7.05(\mathrm{~m}, 2 \mathrm{H}), 7.13-7.21(\mathrm{~m}, 5 \mathrm{H}) .{ }^{13} \mathbf{C}$ NMR (50 MHz) $\delta$ 21.5, 57.8, 61.6, 71.0, 73.0, 75.2, 128.3 (2 C), 129.0 (5 C), 129.2 (2 C), 132.8, 135.1, 144.8. IR (KBr): 3479, 3021, 2925, 2855, 1597, 1494, 1456, 1403, 1324, 1299, 1215, 1149, 1096, 1049, 989, 899, 812, $763 \mathrm{~cm}^{-1}$. MS (ES): $715[2 \mathrm{M}+\mathrm{Na}]^{+}, 369[\mathrm{M}+\mathrm{Na}]^{+}(100 \%), 347[\mathrm{M}+1]^{+}$. Anal. calcd for $\mathrm{C}_{18} \mathrm{H}_{18} \mathrm{O}_{5} \mathrm{~S}: \mathrm{C}, 62.41 ; \mathrm{H}, 5.24 ; \mathrm{S}, 9.26$. Found: C, 62.75; H, 5.09; S, 9.13.

11. Synthesis of $(2 S, 4 S, 5 S)-4-B r o m o-5-h y d r o x y-2-p h e n y l-d i h y d r o-2 H$-pyran-3(4H)-one, $(S)-17$, and $(2 S, 4 R, 5 S)-4-B r o m o-5-h y d r o x y-2-p h e n y l-d i h y d r o-2 H$-pyran-3(4H)-one, $(R)-17$.

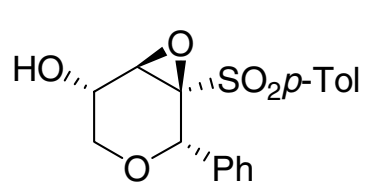

16

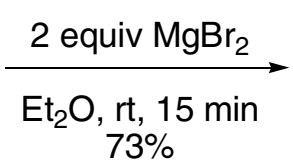
$73 \%$<smiles>O=C1[C@H](c2ccccc2)OC[C@@H](O)[C@H]1Br</smiles>

$(R):(S)-17$

To a solution of sulfone $16(10 \mathrm{mg}, 0.03 \mathrm{mmol})$ in $\mathrm{Et}_{2} \mathrm{O}$ at $\mathrm{rt}$, under an argon atmosphere, a freshly prepared $0.3 \mathrm{M}$ solution of $\mathrm{MgBr}_{2}\left(0.5 \mathrm{~mL}, 0.50 \mathrm{mmol}, 5.0\right.$ equiv) in $\mathrm{Et}_{2} \mathrm{O}$ was added. The mixture was stirred at $\mathrm{rt}$, monitored by TLC until completion (15 min) and then quenched with a saturated solution of $\mathrm{NH}_{4} \mathrm{Cl}(3 \mathrm{~mL} / \mathrm{mmol})$ and water $(3 \mathrm{~mL} / \mathrm{mmol})$. The layers were separated and the aqueous phase was extracted with EtOAc $(5 \mathrm{~mL} / \mathrm{mmol}, 3$ times). The combined organic extracts were washed with a saturated solution of $\mathrm{NaCl}$, dried over anhydrous $\mathrm{MgSO}_{4}$, filtered and concentrated. The ${ }^{1} \mathrm{H}$ NMR spectra of the crude product showed a 70:30 mixture of (4S)-17 and (4R)-17. Purification by chromatography (20-50\% EtOAc-hexane) afforded $6 \mathrm{mg}(0.022 \mathrm{mmol}, 73 \%)$ of a 60:40 mixture of (4S)-17 and (4R)-17. Recrystallization of the crude product from EtOAc-Et ${ }_{2} \mathrm{O}$ gave $3 \mathrm{mg}$ of (4S)-17 as a white solid. After 2 days in $\mathrm{CDCl}_{3}$ epimerization at $\mathrm{C}-4$ took place affording a 60:40 mixture of diastereomers.

Data for (4S)-17: $\quad \boldsymbol{R}_{f}=0.30$ (40\% EtOAc-hexane). ${ }^{1} \mathbf{H}$ NMR (200 MHz) $\delta 2.61$ (br s, $1 \mathrm{H}$, $\mathrm{OH}), 4.15$ (dd, $1 \mathrm{H}, J=12.6,1.6 \mathrm{~Hz}, \mathrm{H}-2), 4.36$ (dd, $1 \mathrm{H}, J=12.6,2.7 \mathrm{~Hz}, \mathrm{H}-2), 4.56$ (m, $1 \mathrm{H}, \mathrm{H}-3)$, 5.05 (s, $1 \mathrm{H}, \mathrm{H}-6), 5.11$ (d, $1 \mathrm{H}, J=3.3 \mathrm{~Hz}, \mathrm{H}-4), 7.26-7.38$ (m, $5 \mathrm{H})$.

Partial data for (4R)-17 (from the mixture): $\boldsymbol{R}_{f}=0.28$ (40\% EtOAc-hexane). ${ }^{\mathbf{1}} \mathbf{H}$ NMR (200 MHz) $\delta 2.70$ (br s, $1 \mathrm{H}, \mathrm{OH}), 5.44$ (s, $1 \mathrm{H}, \mathrm{H}-6), 5.11$ (d, $1 \mathrm{H}, J=3.3 \mathrm{~Hz}, \mathrm{H}-4), 7.26-7.38$ (m, 5 H). IR (film): 3369, 3057, 3021, 2924, 2847, 1740, 1700, 1606, 1494, 1447, 1125, 1095, 1028, 751, 698 $\mathrm{cm}^{-1}$. 
12. Synthesis of (+)-(2S,3S,5R)-2-Phenyl-tetrahydro-2H-pyran-3,5-diol, 18.<smiles>O=C1[C@H](c2ccccc2)OC[C@@H](O)[C@@H]1Br</smiles>

$(R):(S)-17$

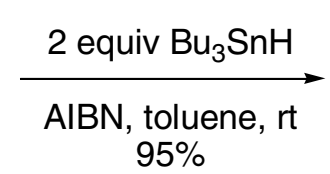

$95 \%$

To a solution of a 60:40 mixture of $(4 S)$ and $(4 R)-17(6 \mathrm{mg}, 0.022 \mathrm{mmol})$ in toluene (10 $\mathrm{mL} / \mathrm{mmol}$ ) at $\mathrm{rt}$, under an argon atmosphere, was added tributyltin hydride (2 equiv, $14 \mu \mathrm{L}, 0.044$ mmol) and a catalytic amount of AIBN. The mixture was stirred at rt and monitored by TLC until completion $(2 \mathrm{~h})$ and the solvent was removed under reduced pressure. Purification by chromatography (50-80\% EtOAc-hexane) afforded $4 \mathrm{mg}(0.021 \mathrm{mmol}, 95 \%)$ of diol 18 as a colorless oil.

Data for 18: $\boldsymbol{R}_{f}=0.18\left(80 \%\right.$ EtOAc-hexane). $[\alpha]^{20}{ }_{\mathbf{D}}=+28.7(c=0.18) .{ }^{1} \mathbf{H}$ NMR (300 MHz) $\delta 1.50(\mathrm{~d}, 1 \mathrm{H}, J=2.9 \mathrm{~Hz}, \mathrm{OH}), 1.73(\mathrm{ddd}, 1 \mathrm{H}, J=13.5,10.7,3.2 \mathrm{~Hz}, \mathrm{H}-4), 1.98(\mathrm{~d}, 1 \mathrm{H}, J=6.8 \mathrm{~Hz}$, OH), 2.39 (ddt, $1 \mathrm{H}, J=13.2,4.6,2.9 \mathrm{~Hz}, \mathrm{H}-4), 3.71$ (dd, $1 \mathrm{H}, J=12.2,1.2 \mathrm{~Hz}, \mathrm{H}-6), 3.94$ (m, 1 H, H3), 3.98 (s, 1 H, H-2), 4.00 (m, 1 H, H-6), 4.10 (m, 1 H, H-5), 7.30-7.44 (m, 5 H). ${ }^{13}$ C NMR (50 MHz) $\delta 38.6$ (C-4), 67.3, 67.8, 72.8, 85.6, 127.4 (2 C), 128.7, 128.8 (2 C). IR (film): 3338, 2956, 2925, 2847, 1450, 1346, 1261, 1107, 1048, 987, 903, 757, $700 \mathrm{~cm}^{-1}$. MS (ES): $217[\mathrm{M}+\mathrm{Na}]^{+}(100 \%), 177$ $[(\mathrm{M}-18)+1]^{+}$.

13. Synthesis of (-)-(2S)-[(2S,3R,4R,5S)-2-Phenyl-3-(p-tolylsulfonyl)-3,4-epoxy-tetrahydro- $2 H$ pyran-3-yl]-2-methoxy-2-phenylacetate, 19 , and $(2 R)-[(2 S, 3 R, 4 R, 5 S)-2$-Phenyl-3-(p-tolylsulfonyl)3,4-epoxy-tetrahydro-2H-pyran-3-yl]-2-methoxy-2-phenylacetate, 20.

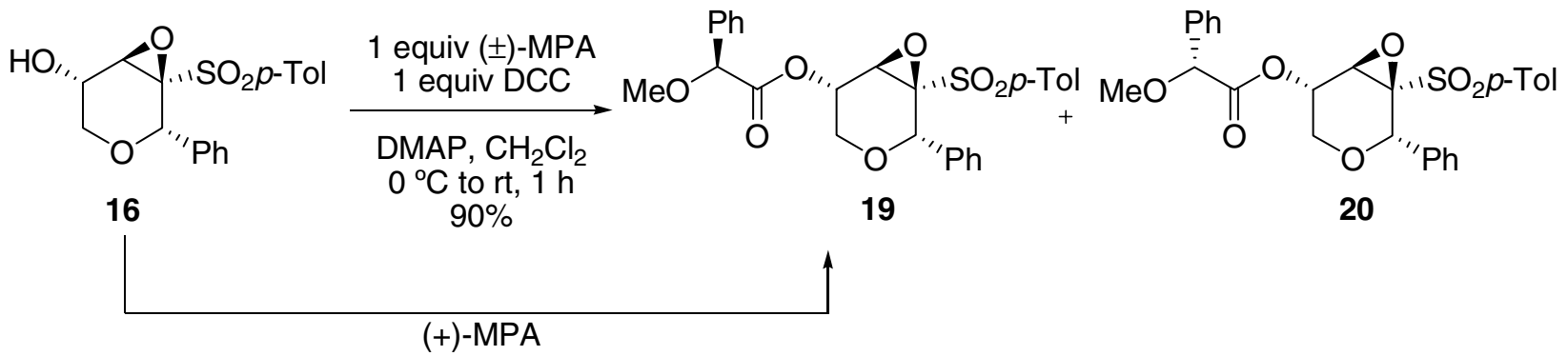

To a solution of sulfone 16 (3 $\mathrm{mg}, 0.009 \mathrm{mmol})$ and 1.1 equiv of $( \pm)$-2-methoxy-2-phenylacetic acid $(1.7 \mathrm{mg}, 0.010 \mathrm{mmol})$ in $\mathrm{CH}_{2} \mathrm{Cl}_{2}(10 \mathrm{~mL} / \mathrm{mmol})$ at $0{ }^{\circ} \mathrm{C}$, was added dicyclohexylcarbodiimide (1.1 equiv, $2 \mathrm{mg}, 0.010 \mathrm{mmol}$ ) and a catalytic amount of DMAP. The mixture was allowed to warm to room temperature and monitored by TLC until starting material disappearance $(3 \mathrm{~h})$. The reaction 
mixture was filtered to remove dicyclohexylurea and the ester was purified by chromatography $(20 \%$ EtOAc-hexane) to give $4 \mathrm{mg}(0.008 \mathrm{mmol}, 90 \%)$ of a 50:50 mixture of $\mathbf{1 9}$ and $\mathbf{2 0 .}$

In an identical experiment with (+)-2-methoxy-2-phenylacetic acid, $4 \mathrm{mg}(0.008 \mathrm{mmol}, 90 \%)$ of 19 was obtained as a white solid. The ${ }^{1} \mathrm{H}$ NMR of the crude product did not show any signal of compound $\mathbf{2 0}$ and thus the optical purity of $\mathbf{1 6}$ was established.

Data for 19: $\boldsymbol{R}_{f}=0.15\left(30 \%\right.$ EtOAc-hexane). $[\alpha]^{20}{ }_{\mathbf{D}}=-44.1(c=0.17) .{ }^{1} \mathbf{H}$ NMR (300 MHz) $\delta 2.29$ (s, 3 H, Me-p-Tol), 3.44 (s, 3 H, MeO), 3.67 (dd, 1 H, J =13.2, 3.8 Hz, H-6), 3.89 (dd, 1 H, J = 13.2, $2.4 \mathrm{~Hz}, \mathrm{H}-6), 4.34$ (d, $1 \mathrm{H}, J=4.6 \mathrm{~Hz}, \mathrm{H}-4), 4.86$ (s, $\left.1 \mathrm{H}, \mathrm{H}_{\alpha}\right), 5.11$ (m, $\left.1 \mathrm{H}, \mathrm{H}-5\right), 5.24$ (s, $1 \mathrm{H}$, H-2), 6.91 (d, 2 H, J = 8.3 Hz), 7.02 (m, 1 H), 7.03 (d, 2 H, J = 7.3 Hz), 7.10-7.17 (m, 4 H), 7.31-7.39 (m, 3 H), 7.47-7.51 (m, 2 H). IR (film): 3065, 2963, 2927, 1750, 1591, 1494, 1455, 1320, 1260, 1111, $801 \mathrm{~cm}^{-1}$. MS (ES): $517[\mathrm{M}+\mathrm{Na}]^{+}(100 \%), 495\left[(\mathrm{M}+1]^{+}\right.$.

Partial data for $\mathbf{2 0}$ (from the mixture): $\boldsymbol{R}_{f}=0.22$ (30\% EtOAc-hexane). ${ }^{1} \mathbf{H}$ NMR (300 MHz) $\delta 2.29$ (s, 3 H, Me-p-Tol), 3.44 (s, 3 H, MeO), 3.58 (dd, 1 H, J =13.2, 3.8 Hz, H-6), 3.71 (dd, 1 H, $J=$ 13.2, $2.4 \mathrm{~Hz}, \mathrm{H}-6), 4.50$ (d, $1 \mathrm{H}, J=4.4 \mathrm{~Hz}, \mathrm{H}-4), 4.88$ (s, $\left.1 \mathrm{H}, \mathrm{H}_{\alpha}\right), 5.11$ (m, $\left.1 \mathrm{H}, \mathrm{H}-5\right), 5.26$ (s, $1 \mathrm{H}$, H-2).

\section{Structural assignments.}

\subsection{Relative stereochemistry.}

The relative cis-trans stereochemistry between the substituents at C-2 and C-3 in dihydropyran 5b was derived from the sulfoxide-sulfenate adduct $\boldsymbol{e n t - 6 b}$ considering complete transfer of chirality of the rearrangement. The relative stereochemistry of ent-6b was established by synthesis of the acetate $\mathbf{7 b}$ and the corresponding Mitsunobu-inversion product $\mathbf{8 b}$ (figure 1) and comparison of their spectral features. The coupling constants between $\mathrm{H}_{3}-\mathrm{H}_{2 \mathrm{eq}}$ and $\mathrm{H}_{3}-\mathrm{H}_{2 \mathrm{x}}$ establish a pseudoaxial arrangement for $\mathrm{H}_{3}$ in $\mathbf{7 b}\left(J_{3-2 \mathrm{ax}}=5.1 \mathrm{~Hz}, J_{3-2 \mathrm{eq}}=4.4 \mathrm{~Hz}\right)$ and a pseudoequatorial arrangement for $\mathrm{H}_{3}$ in $8 \mathbf{b}\left(J_{3-2 \mathrm{ax}}=2.6 \mathrm{~Hz}, J_{3-2 \mathrm{eq}}<1 \mathrm{~Hz}\right)$. 


\section{Figure 1}

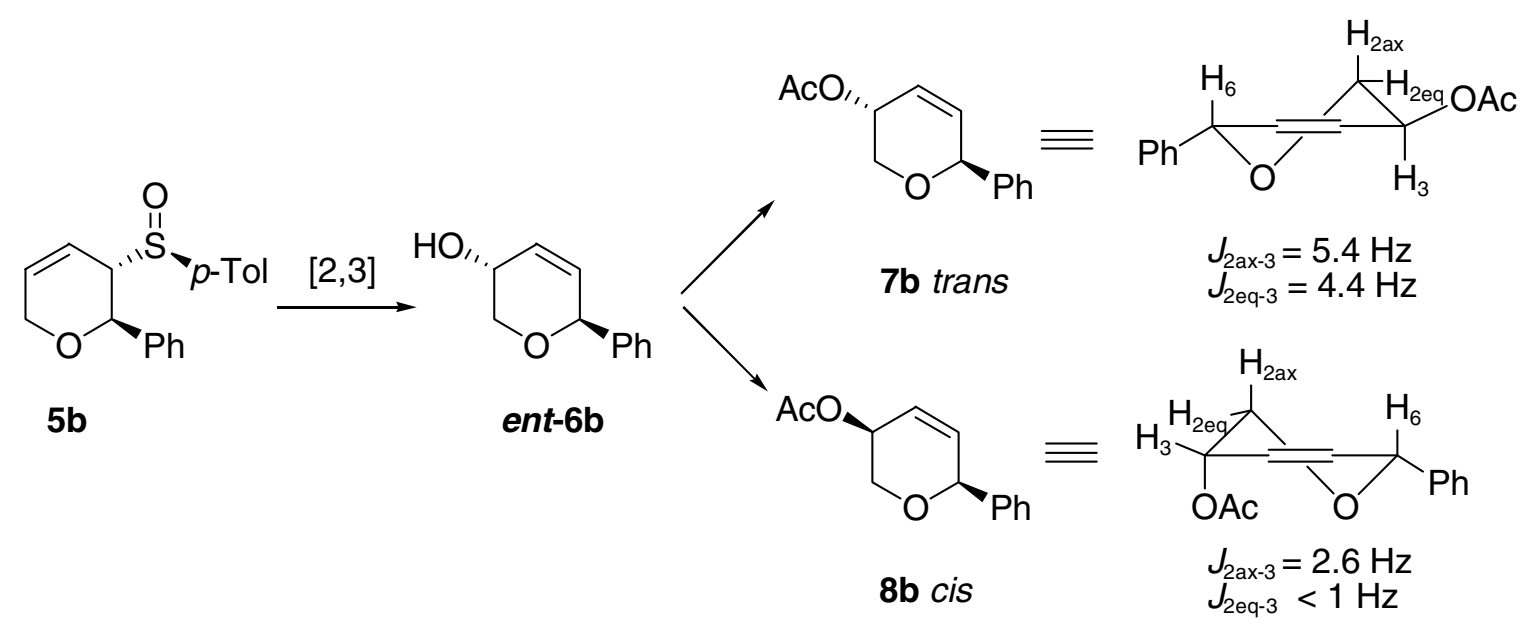

\subsection{Absolute stereochemistry.}

To determine the absolute stereochemistry of epoxy sulfone 16 we prepared the corresponding $(S)$ and $(R)$-methoxy phenyl acetates 19 and 20. Previous studies suggest that for the $\mathrm{L}_{2} \mathrm{~L}_{1} \mathrm{CH}-\mathrm{CO}$ $\mathrm{CHPh}-\mathrm{OMe}$ fragment, the preferred conformer in each case is that where the $\mathrm{C}_{\alpha}-\mathrm{OMe}$ bond, the $\mathrm{C}=\mathrm{O}$ and the $\mathrm{C}_{5}-\mathrm{H}_{5}$ bond are nearly eclipsed. ${ }^{3}$ As shown in figure $2, \mathrm{H}_{4}$ is further upfield in the $(S)$ isomer 19 due to the shielding effect of the phenyl group. In contrast, in the $(R)$ isomer 20 the phenyl group is shielding $\mathrm{H}_{6 \mathrm{eq}}$.

\section{Figure 2}

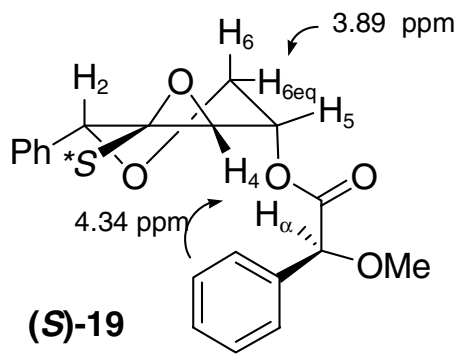

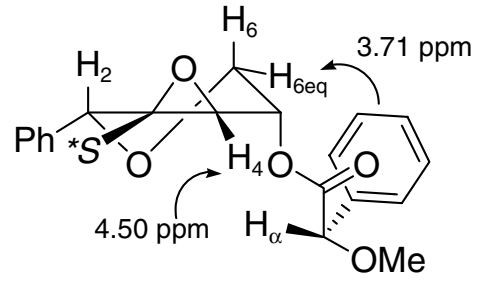

$(R)-20$

Once configuration at C-3 was established, the assignment for C-4 in diol $\mathbf{1 2}$ was made considering the syn stereochemical course of the osmium catalyzed dihydroxylation (figure 3). Finally the configuration at C-5 was based on the coupling constant between $\mathrm{H}_{5}$ and $\mathrm{H}_{4}\left(J_{5-4}=10.1 \mathrm{~Hz}\right)$ which is in agreement with a trans relationship between the substituents at C-4 and C-5.

Figure 3

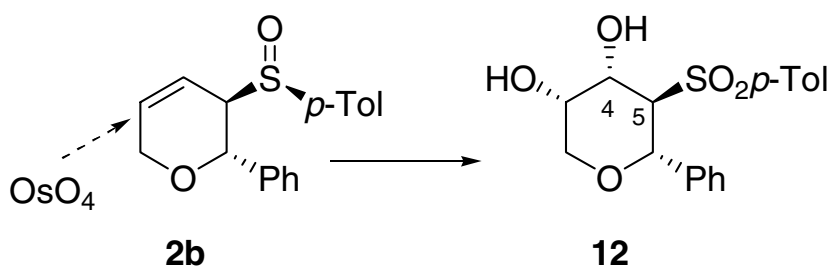




\subsection{Assignments for dihydropyrans $2 a-e, 4 a$ and $5 a$.}

For all dihydropyrans 2a-e the assignment was established by assuming a similar stereochemical course of the process and by comparison of their spectral features with those of compound $\mathbf{2 b}$. The absolute configuration for $\mathbf{5 b}$ was determined by oxidation to the sulfone $\boldsymbol{e n t} \mathbf{- 9 b}$, identical to 9b except for the optical rotation (figure 4). Moreover, the sulfoxide-sulfenate rearrangement of $\mathbf{5 b}$ afforded $\boldsymbol{e n t} \mathbf{- 6} \mathbf{b}$, enantiomer of that derived from $\mathbf{2 b}$.

Figure 4

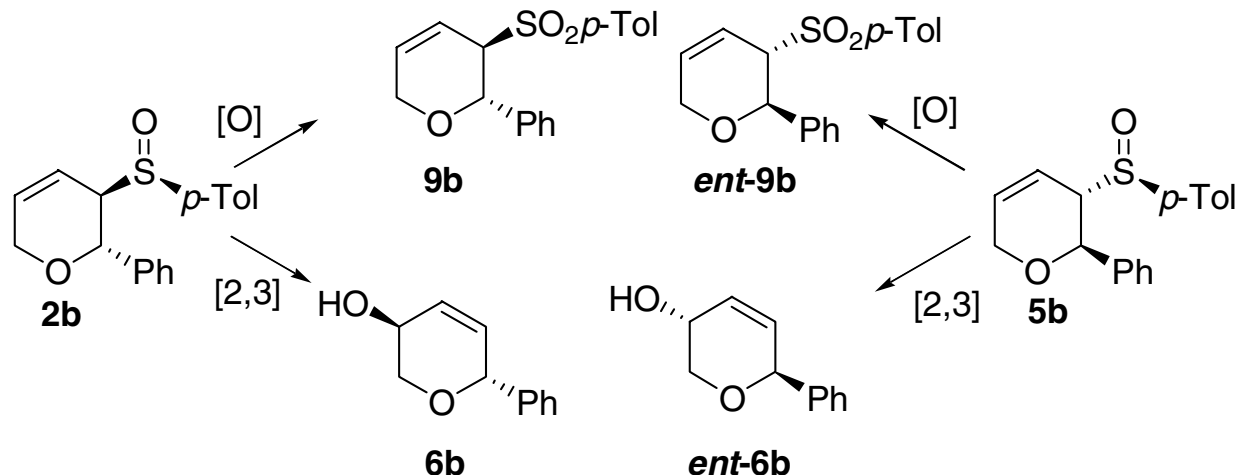

In contrast, the oxidation of $\mathbf{4 a}$ gave sulfone 10a, different to that obtained from 2a (figure 5). This result is in agreement with a relative cis relationship between the substituents at C-3 and C-2 in compound 4a.

\section{Figure 5}

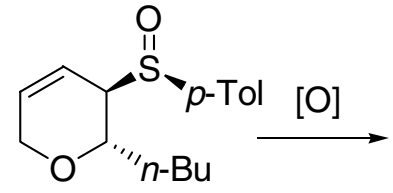

2a<smiles>CCCCC1OCC=CC1S(=O)(=O)O[Na]</smiles>

$9 a$

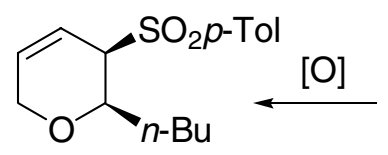

$10 a$<smiles>CCCCC1OCC=CC1S(=O)[O+][Ca]</smiles>

$4 a$

In many cases these assignments were supported by NOESY-1D experiments. The preferred conformer in each case was based on the coupling constant between $\mathrm{H}_{2}$ and $\mathrm{H}_{3}$ (figure 6). 


\section{Figure 6}<smiles>[O]S(=O)C1C=CCO[C@@H]1c1ccccc1</smiles>

2b

$J_{2-3}=2.9 \mathrm{~Hz}$<smiles>CCCCC1OCC=CC1S(=O)O[Ba]</smiles>

2a

$J_{2-3}=2-3 \mathrm{~Hz}$
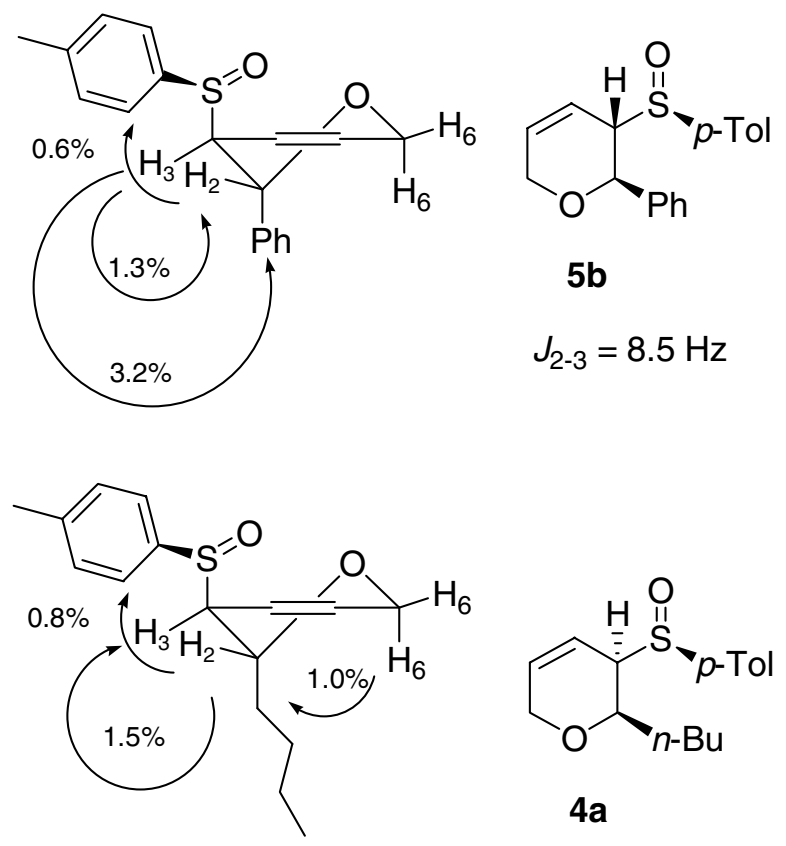

$J_{2-3}=2.2 \mathrm{~Hz}$

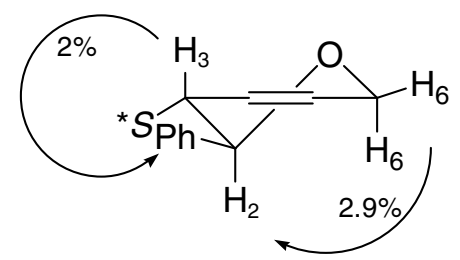

$J_{2-3}=8.5 \mathrm{~Hz}$

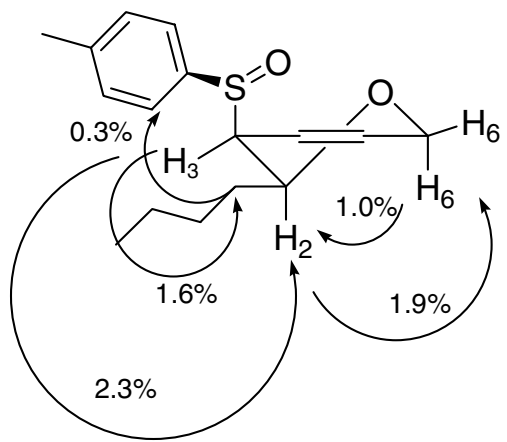

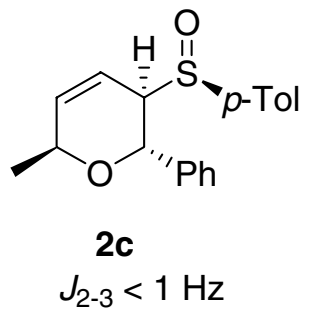

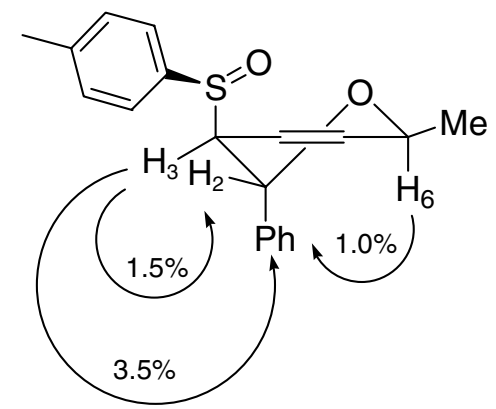

\section{References.}

1. Watson, S. C.; Eastham, J. E. J. Organomet. Chem. 1967, 9, 165-168. In some cases this procedure was not completely accurate particularly for "old" bottles of reagent. Then, the reagent was titrated by reaction with a slight excess of 3,4-dimethoxybenzaldehyde in THF, standard work-up and measuring accurate integrals in the ${ }^{1} \mathrm{H}$ NMR of the crude reaction mixture.

2. Westwell, A. D.; Thorton-Pett, M.; Rayner, C. M. J. Chem. Soc. Perkin Trans 1 1995, 847-859.

3. Latypov, S. K.; Seco, J. M.; Quiñoá, E.; Riguera, R. J. Org. Chem. 1996, 61, 8569-8577. 